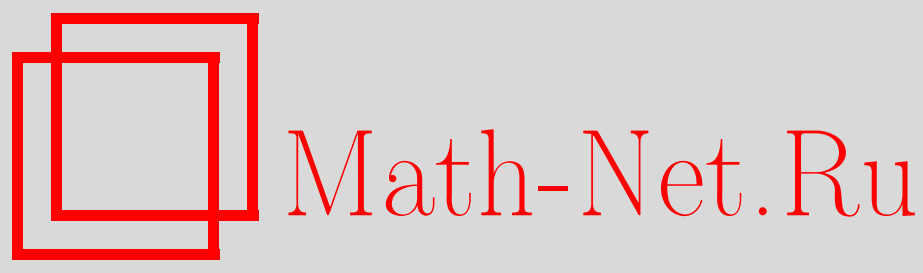

А. В. Михайлов, Дж. П. Ванг, П. Ксенитидис, Рекурсионные операторы, законы сохранения и условия интегрируемости для разностных уравнений, ТМФ, 2011, том 167, номер 1, 23-49

DOI: https://doi.org/10.4213/tmf6624

Использование Общероссийского математического портала Math-Net.Ru подразумевает, что вы прочитали и согласны с пользовательским соглашением http: //www . mathnet.ru/rus/agreement

Параметры загрузки:

IP : 54.147 .182 .235

26 апреля 2023 г., 18:17:19

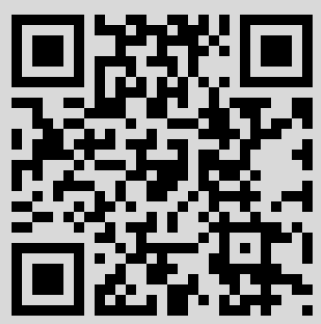




\title{
РЕКУРСИОННЫЕ ОПЕРАТОРЫ, ЗАКОНЫ СОХРАНЕНИЯ И УСЛОВИЯ ИНТЕГРИРУЕМОСТИ ДЛЯ РАЗНОСТНЫХ УРАВНЕНИЙ
}

\begin{abstract}
Предпринята попытка предложить алгебраический подход к теории интегрируемых разностных уравнений. Определено понятие рекурсионного оператора и показано, что он порождает бесконечную последовательность симметрий и канонических законов сохранения для разностного уравнения. Как и в случае дифференциальных уравнений в частных производных, эти канонические плотности могут играть роль условий интегрируемости для разностных уравнений. Получены рекурсионные операторы для уравнения Виале и всех уравнений Адлера-Бобенко-Суриса.
\end{abstract}

Ключевые слова: разностные уравнения, интегрируемость, условия интегрируемости, симметрии, законы сохранения, рекурсионный оператор.

\section{1. ВВЕДЕНИЕ}

Природа интегрируемости дифференциальных уравнений в частных производных хорошо изучена [1]-[3]. Ее можно связать с существованием представлений Лакса, преобразований Беклунда и Миуры, билинейных представлений Хироты, многосолитонных решений, свойства Пенлеве, бигамильтоновых структур, рекурсионных операторов и бесконечных иерархий симметрий и законов сохранения. Несмотря на отсутствие общепризнанного определения интегрируемости, типы уравнений, обладающих некоторыми из перечисленных выше свойств, совпадают. В симметрийном подходе за определение интегрируемости принимается существование бесконечной иерархиии симметрий и законов сохранения. Именно этот подход позволил получить наиболее полные результаты классификации интегрируемых уравнений, основанные на этом свойстве [4]-[10]. В работах [11], [12] было показано, что подход, базирующийся на симметриях, эффективен в задаче о классификации интегрируемых дифференциально-разностных уравнений.

В настоящее время активно развивается сравнительно недавно возникшая теория интегрируемых разностных (или дискретных) уравнений. Эта теория унаследовала

*Applied Mathematics Department, University of Leeds, UK.

E-mail: a.v.mikhailov@gmail.com

${ }^{\dagger}$ School of Mathematics and Statistics, University of Kent, UK 
некоторые методы и подходы из теории интегрируемых дифференциальных уравнений в частных производных (см., например, [13], [14]). В качестве критерия интегрируемости на квадратной решетке (или квад-уравнений) для разностных уравнений было предложено [15], [16] свойство 3D-совместности, или совместности по сторонам куба [17]. Все интегрируемые аффинно-линейные квад-уравнения (удовлетворяющие некоторым дополнительным условиям симметрии относительно отражений решетки) были проклассифицированы Адлером, Бобенко и Сурисом (АБС) [18] (см. также [19]). Авторы этих работ получили список из семи уравнений (с точностью до точечных дробно-линейных преобразований), которые также известны как уравнения АБС. К сожалению, метод, основанный на свойстве многомерной совместности, нельзя непосредственно применить к заданному уравнению на решетке. Действительно, чтобы проверить условие 3D-совместности, необходимо знать уравнения, соответствующие сторонам куба.

Еще одно характеристическое свойство интегрируемого уравнения - это обращение в нуль его алгебраической энтропии [20]. Этим свойством воспользовался Виале [21], чтобы выделить уравнение

$$
\begin{aligned}
Q:= & a_{1} u_{0,0} u_{1,0} u_{0,1} u_{1,1}+a_{2}\left(u_{0,0} u_{1,0} u_{0,1}+u_{1,0} u_{0,1} u_{1,1}+u_{0,1} u_{1,1} u_{0,0}+u_{1,1} u_{0,0} u_{1,0}\right)+ \\
& +a_{3}\left(u_{0,0} u_{1,0}+u_{0,1} u_{1,1}\right)+a_{4}\left(u_{1,0} u_{0,1}+u_{0,0} u_{1,1}\right)+ \\
& +a_{5}\left(u_{0,0} u_{0,1}+u_{1,0} u_{1,1}\right)+a_{6}\left(u_{0,0}+u_{1,0}+u_{0,1}+u_{1,1}\right)+a_{7}=0
\end{aligned}
$$

где $a_{i}$ - произвольные комплексные параметры. С помощью точечного дробно-линейного преобразования уравнение (1) с параметрами в общем положении можно привести к уравнению Адлера, также известному в классификации АБС как уравнение Q4. Поэтому неудивительно, что уравнение Виале (1) имеет иерархию симметрий [22]. Все уравнения АБС можно получить из уравнения Виале простой конкретизацией параметров, и это удобный способ представления уравнений АБС. Очевидным преимуществом подхода Виале является возможность его приложения к заданному уравнению.

Цель настоящей работы состоит в развитии теории разностных уравнений, основанной на изучении симметрий, законов сохранения и (формальных) рекурсионных операторов. Под интегрируемостью разностных уравнений мы понимаем существование бесконечной иерархии его симметрий. В настоящей работе мы рассматриваем квад-уравнения $Q=0$, где предполагается, что $Q$ - неприводимый аффинно-линейный многочлен над полем $\mathbb{C}$. Симметрии, законы сохранения и в действительности все рациональные функции, которые имеют смысл на решениях разностного уравнения, являются элементами разностного поля отношений $\mathcal{F}_{Q}$, которое определяется в разделе 2 . Аффинная линейность уравнения позволяет ввести отображение исключения $\mathcal{E}$, которое редуцирует любой элемент поля $\mathcal{F}_{Q}$ к рациональной функции динамических переменных. Это отображение весьма полезно при вычислениях и доказательствах, поскольку две функции $f$ и $g$ решеточных переменных эквивалентны по модулю разностного уравнения тогда и только тогда, когда $\mathcal{E}(f)=\mathcal{E}(g)$. В разделе 2 представлен замкнутый набор основных определений, которые нам потребуются в настоящей работе.

В разделе 3 дается определение рекурсионного оператора для разностных уравнений. Рекурсионный оператор, действующий на затравочную симметрию разностно- 
го уравнения, порождает бесконечную иерархию симметрий этого разностного уравнения. В работе показано, что вычеты степеней рекурсионного оператора являются (каноническими) плотностями законов сохранения для соответствующих разностных уравнений. Таким образом можно породить бесконечную последовательность канонических законов сохранения (одновременно плотностей и потоков). Как и в дифференциальном случае (см. [4]-[6]), эти канонические законы сохранения можно использовать как условия интегрируемости для разностного уравнения. Они являются необходимыми условиями существования (формального) рекурсионного оператора и, в конечном счете, бесконечной иерархии симметрий разностного уравнения.

В разделе 4 представлены рекурсионные операторы для уравнения Виале и всех уравнений АБС. Эти рекурсионные операторы порождают бесконечные иерархии симметрий для каждого уравнения АБС. Соответствующие канонические законы сохранения дают бесконечную последовательность плотностей и соответствующих потоков возрастающего порядка. Первый член в этой последовательности для всех уравнений АБС был получен в работе [23] путем прямого вычисления. Недавно было показано, что метод Гарднера, используемый для интегрируемых дифференциальных уравнений в частных производных, можно адаптировать к разностным уравнениям, и он был применен для построения бесконечной последовательности законов сохранения для уравнения Н1, а также некоторых других уравнений, включая несимметричные [24], [25].

Данное исследование было отчасти стимулировано результатами, полученными Леви и Ямиловым в работе [26], где они начали развивать теорию интегрируемых разностных уравнений, основанную на изучении их симметрий. Цель теории Леви и Ямилова состоит в классификации разностных уравнений, обладающих симметриями. Они добились значительных успехов в формализации теории и коснулись проблемы динамических переменных. Леви и Ямилов получили одно условие интегрируемости для разностных уравнений, которое совпадает с первым элементом нашей бесконечной последовательности канонических законов сохранения. Их подход основан на изучении симметрий и не использует концепцию (формального) рекурсионного оператора.

Подчеркнем, что наше допущение о том, что разностное уравнение является аффинно-линейным многочленом, а его симметрии представляют собой рациональные функции, сделано по большей части для ясности изложения; оно вполне подходит к случаю уравнений АБС. От этого допущения можно избавиться при некоторых несущественных технических условиях, гарантирующих, что разностное уравнение имеет единственное решение для открытого множества начальных данных. Результаты настоящей работы можно перенести на случай локально аналитических функций решеточных переменных.

\section{2. ОСНОВНЫЕ ОПРЕДЕЛЕНИЯ}

В данном разделе мы вводим отображение исключения $\mathcal{E}$ и определяем динамические переменные для аффинно-линейных разностных квад-уравнений. Мы также приводим основные определения симметрий и законов сохранения для разностных уравнений. 
2.1. Разностные уравнения и динамические переменные. Разностные уравнения на пространстве $\mathbb{Z}^{2}$ можно рассматривать как дискретный аналог дифференциальных уравнений в частных производных. Обозначим через $u=u(n, m)$ комплекснозначную функцию $u: \mathbb{Z}^{2} \mapsto \mathbb{C}$, где $n$ и $m$ - "независимые" переменные, а $u$ играет роль “зависимой” переменной в разностном уравнении. Вместо частных производных мы имеем два коммутирующих отображения сдвига $\mathcal{S}$ и $\mathcal{T}$, которые определяются как

$$
\begin{aligned}
\mathcal{S}: & u \mapsto u_{1,0}=u(n+1, m), \\
\mathcal{T}: & u \mapsto u_{0,1}=u(n, m+1), \\
\mathcal{S}^{p} \mathcal{T}^{q}: & u \mapsto u_{p, q}=u(n+p, m+q) .
\end{aligned}
$$

Для единообразия удобно обозначить исходную функцию $u$ через $u_{0,0}$. В теории разностных уравнений мы будем рассматривать $u_{p, q}$ как переменные. Множество всех сдвигов переменной $u$ обозначим через $U=\left\{u_{p, q} \mid(p, q) \in \mathbb{Z}^{2}\right\}$. Для функции $f=f\left(u_{p_{1}, q_{1}}, \ldots, u_{p_{k}, q_{k}}\right)$ переменных $u_{p, q}$ действие операторов $\mathcal{S}$ и $\mathcal{T}$ определяется KaK

$$
\mathcal{S}^{i} \mathcal{T}^{j}(f)=f_{i, j}=f\left(u_{p_{1}+i, q_{1}+j}, \ldots, u_{p_{k}+i, q_{k}+j}\right) .
$$

Разностное квад-уравнение можно определить как

$$
Q\left(u_{0,0}, u_{1,0}, u_{0,1}, u_{1,1}\right)=0
$$

где $Q\left(u_{0,0}, u_{1,0}, u_{0,1}, u_{1,1}\right)$ - неприводимый многочлен по “зависимой” переменной $u=u_{0,0}$ и ее сдвигам. Неприводимость означает, что многочлен $Q$ нельзя разбить на множители, т. е. представить в виде произведения двух многочленов. Предполагается, что уравнение (2) справедливо в каждой точке $(n, m) \in \mathbb{Z}^{2}$ и, таким образом, представляет бесконечную систему уравнений

$$
Q_{p, q}=Q\left(u_{p, q}, u_{p+1, q}, u_{p, q+1}, u_{p+1, q+1}\right)=0, \quad(p, q) \in \mathbb{Z}^{2} .
$$

В настоящей работе мы рассмотрим только полиномиальные $Q$. Это ограничение не очень существенно для нашего построения, однако оно позволяет сделать изложение более наглядным. Более того, при рассмотрении квад-уравнений мы будем предполагать, что неприводимый аффинно-линейный многочлен $Q$ нетривиально зависит от всех переменных, т. е.

$$
\frac{\partial Q}{\partial u_{i, j}} \neq 0, \quad \frac{\partial^{2} Q}{\partial u_{i, j}^{2}}=0, \quad i, j \in\{0,1\}, \quad Q \in \mathbb{C}\left[u_{0,0}, u_{1,0}, u_{0,1}, u_{1,1}\right] .
$$

Пусть $\mathbb{C}[U]$ - кольцо многочленов по переменным $U$, при этом считается, что каждый многочлен имеет конечное число членов. Отображения $\mathcal{S}$ и $\mathcal{T}$ являются автоморфизмами $\mathbb{C}[U]$, и, таким образом, $\mathbb{C}[U]$ является разностным кольцом. Обозначим через $\hat{J}_{Q}=\left\langle\left\{Q_{p, q}\right\}\right\rangle$ идеал, порожденный разностным уравнением и всеми его сдвигами (3). Для любого $a \in \hat{J}_{Q}$ имеем $\mathcal{S}(a) \in \hat{J}_{Q}$ и $\mathcal{T}(a) \in \hat{J}_{Q}$. Таким образом, $\hat{J}_{Q}$ является разностным идеалом. Если идеал $\hat{J}_{Q}$ является простым, то мы будем опускать шляпку и обозначать его $J_{Q}$. В противном случае в качестве $J_{Q}$ мы берем его алгебраическое насыщение $J_{Q}=\operatorname{sat}(Q)$ (определение алгебраического 
насыщения $\operatorname{sat}(Q)$ можно найти в работе [27]). Из теоремы 22 работы [27] следует, что для неприводимых аффинно-линейных уравнений разностный идеал $\operatorname{sat}(Q)$ является простым, и, таким образом, разностное факторкольцо $\mathbb{C}[U] / J_{Q}$ является областью целостности.

Рациональные функции переменных $u_{p, q}$, которые можно ограничить на решения разностного уравнения (т. е. знаменатели которых не обращаются в нуль при подстановке решений), образуют поле:

$$
\mathcal{F}_{Q}=\left\{[a] /[b] \mid a, b \in \mathbb{C}[U], b \notin J_{Q}\right\},
$$

где $[a]$ обозначает класс эквивалентных многочленов (два многочлена $f, g \in \mathbb{C}[U]$ считаются эквивалентными, что обозначается как $f \equiv g$, если $f-g \in J_{Q}$ ). Для $a, b, c, d \in \mathbb{C}[U], b, d \notin J_{Q}$, две рациональные функции $a / b$ и $c / d$ представляют один и тот же элемент $\mathcal{F}_{Q}$, если $a d-b c \in J_{Q}$. Такие рациональные функции мы будем называть эквивалентными и обозначать $a / b \equiv c / d$. Значения эквивалентных рациональных функций на всех решениях разностного уравнения совпадают.

Поля рациональных функций по переменным

$$
U_{\mathbf{s}}=\left\{u_{n, 0} \mid n \in \mathbb{Z}\right\}, \quad U_{\mathbf{t}}=\left\{u_{0, n} \mid n \in \mathbb{Z}\right\}, \quad U_{0}=U_{\mathbf{s}} \cup U_{\mathbf{t}}
$$

обозначаются как

$$
\mathcal{F}_{\mathbf{s}}=\mathbb{C}\left(U_{\mathbf{s}}\right), \quad \mathcal{F}_{\mathbf{t}}=\mathbb{C}\left(U_{\mathbf{t}}\right), \quad \mathcal{F}_{0}=\mathbb{C}\left(U_{0}\right)
$$

В общем случае работа с многочленами и рациональными функциями по модулю идеала представляет собой весьма трудоемкую вычислительную задачу. В нашем случае идеал $J_{Q}$ порождается аффинно-линейными многочленами $Q_{p, q}$ и мы можем единственным образом разрешить уравнение $Q=0$ относительно каждой переменной:

$$
\begin{array}{ll}
u_{0,0}=F\left(u_{1,0}, u_{0,1}, u_{1,1}\right), & u_{1,0}=G\left(u_{0,0}, u_{0,1}, u_{1,1}\right), \\
u_{0,1}=H\left(u_{0,0}, u_{1,0}, u_{1,1}\right), & u_{1,1}=M\left(u_{0,0}, u_{1,0}, u_{0,1}\right) .
\end{array}
$$

Поскольку $Q$ - аффинно-линейный многочлен, функции $F, G, H$ и $M$ являются рациональными функциями своих аргументов. Уравнения (4) позволяют рекуррентно и единственным образом выразить любую переменную $u_{p, q}$ в терминах переменных $U_{0}=U_{\mathbf{s}} \cup U_{\mathbf{t}}$.

ОПРЕДЕЛЕНИЕ 1. Для элементов из множества $U$ отображение исключения $\mathcal{E}: U \mapsto \mathbb{C}\left(U_{0}\right)$ рекуррентно определяется следующим образом:

$$
\begin{array}{lll}
\forall p \in \mathbb{Z}, & \mathcal{E}\left(u_{0, p}\right)=u_{0, p}, \quad \mathcal{E}\left(u_{p, 0}\right)=u_{p, 0}, \\
p>0, & q>0, & \mathcal{E}\left(u_{p, q}\right)=M\left(\mathcal{E}\left(u_{p-1, q-1}\right), \mathcal{E}\left(u_{p, q-1}\right), \mathcal{E}\left(u_{p-1, q}\right)\right), \\
p<0, \quad q>0, & \mathcal{E}\left(u_{p, q}\right)=H\left(\mathcal{E}\left(u_{p, q-1}\right), \mathcal{E}\left(u_{p+1, q-1}\right), \mathcal{E}\left(u_{p+1, q}\right)\right), \\
p>0, \quad q<0, & \mathcal{E}\left(u_{p, q}\right)=G\left(\mathcal{E}\left(u_{p-1, q}\right), \mathcal{E}\left(u_{p-1, q+1}\right), \mathcal{E}\left(u_{p, q+1}\right)\right), \\
p<0, & q<0, & \mathcal{E}\left(u_{p, q}\right)=F\left(\mathcal{E}\left(u_{p+1, q}\right), \mathcal{E}\left(u_{p, q+1}\right), \mathcal{E}\left(u_{p+1, q+1}\right)\right) .
\end{array}
$$

Для многочленов $f\left(u_{p_{1}, q_{1}}, \ldots, u_{p_{k}, q_{k}}\right) \in \mathbb{C}[U]$ отображение исключения $\mathcal{E}: \mathbb{C}[U] \mapsto$ $\mathbb{C}\left(U_{0}\right)$ определяется как

$$
\mathcal{E}: f\left(u_{p_{1}, q_{1}}, \ldots, u_{p_{k}, q_{k}}\right) \mapsto f\left(\mathcal{E}\left(u_{p_{1}, q_{1}}\right), \ldots, \mathcal{E}\left(u_{p_{k}, q_{k}}\right)\right) \in \mathbb{C}\left(U_{0}\right) .
$$


Для рациональных функций $a / b, a, b \in \mathbb{C}[U], b \notin J_{Q}$, отображение исключения $\mathcal{E}$ определяется как

$$
\mathcal{E}: \frac{a}{b} \mapsto \frac{\mathcal{E}(a)}{\mathcal{E}(b)} .
$$

Переменные $U_{0}$ мы будем называть динамическими переменными. Легко видеть, что $\mathcal{E}\left(u_{p, q}\right)$, где $p \neq 0$ и $q \neq 0$, есть рациональная функция $|p|+|q|+1$ динамических переменных

$$
\mathcal{E}\left(u_{p, q}\right) \in \mathbb{C}\left(\left\{u_{n, 0}, u_{0, m}|0 \leqslant| n-p|\leqslant| p|, 0 \leqslant| m-q|\leqslant| q \mid\right\}\right) .
$$

Выбор множества динамических переменных не является единственным. Другой популярный выбор - так называемый лестничный набор $\left\{u_{n, n}, u_{n, n+1} \mid n \in \mathbb{Z}\right\}[28]$, который мы в настоящей работе не используем.

Отображение исключения $\mathcal{E}: \mathbb{C}[U] \mapsto \mathbb{C}\left(U_{0}\right)$ является гомоморфизмом разностных колец, его ядро есть $J_{Q}$, а его образ изоморфен как разностное кольцо факторкольцу $\mathbb{C}[U] / J_{Q}$. Поле $\mathbb{C}\left(U_{0}\right)$ является разностным полем с автоморфизмами $\mathcal{E} \circ \mathcal{S}$ и $\mathcal{E} \circ \mathcal{T}$. Отображение исключения $\mathcal{E}: \mathcal{F}_{Q} \mapsto \mathbb{C}\left(U_{0}\right)$ является изоморфизмом разностного поля. Отображение исключения является полезным инструментом, позволяющим установить, что две рациональные функции $f, g$ переменных $U$ эквивалентны (т. е. представляют один и тот же элемент из $\mathcal{F}_{Q}$ ), действительно, $f \equiv g$ тогда и только тогда, когда $\mathcal{E}(f)=\mathcal{E}(g)$. В дальнейшем мы будем использовать логарифмы рациональных функций. Действие $\mathcal{E}$ на $\ln a, a \in \mathcal{F}_{Q}, a \not \equiv 0$, определяется как $\mathcal{E}(\ln a)=\ln (\mathcal{E}(a))$, и, таким образом, мы будем говорить, что $\ln a \equiv \ln b$ тогда и только тогда, когда $\ln (\mathcal{E}(a))=\ln (\mathcal{E}(b))$.

Чтобы проиллюстрировать описанное выше построение, рассмотрим разностное уравнение $Q=0$, где

$$
Q=\left(u_{0,0}-u_{1,1}\right)\left(u_{1,0}-u_{0,1}\right)-\alpha+\beta, \quad \alpha \neq \beta, \quad \alpha, \beta \in \mathbb{C},
$$

известное как уравнение Н1 в классификации АБС или дискретное потенциальное уравнение Кортевега-де Фриза. Предположение, что $\alpha \neq \beta$, необходимо для неприводимости многочлена $Q$. Из определения 1 следует, что

$$
\begin{aligned}
& \mathcal{E}\left(u_{1,1}\right)=M\left(u_{0,0}, u_{1,0}, u_{0,1}\right)=u_{0,0}-\frac{\alpha-\beta}{u_{1,0}-u_{0,1}}, \\
& \mathcal{E}\left(u_{1,2}\right)=\frac{u_{0,1}^{2} u_{0,2}-u_{0,0} u_{0,1}^{2}-(\alpha-\beta) u_{1,0}+u_{0,0} u_{0,1} u_{1,0}-u_{0,1} u_{0,2} u_{1,0}}{\beta-\alpha-u_{0,0} u_{0,1}+u_{0,1} u_{0,2}+u_{0,0} u_{1,0}-u_{0,2} u_{1,0}}, \ldots .
\end{aligned}
$$

Две рациональные функции

$$
f=\frac{u_{0,0}-u_{1,1}}{u_{1,0}-u_{-1,0}}, \quad g=\frac{\alpha-\beta}{\left(u_{1,0}-u_{-1,0}\right)\left(u_{1,0}-u_{0,1}\right)}
$$

эквивалентны, $f \equiv g$, поскольку

$$
\frac{u_{0,0}-u_{1,1}}{u_{1,0}-u_{-1,0}}-\frac{\alpha-\beta}{\left(u_{1,0}-u_{-1,0}\right)\left(u_{1,0}-u_{0,1}\right)}=\frac{Q}{\left(u_{1,0}-u_{-1,0}\right)\left(u_{1,0}-u_{0,1}\right)}
$$

и легко проверить, что $\mathcal{E}(f)=\mathcal{E}(g)=g$. 


\section{2. Симметрии и законы сохранения разностных уравнений.}

ОПредЕЛЕНИЕ 2. Пусть $Q=0$ - разностное уравнение. Тогда $K \in \mathcal{F}_{Q}$ называется генератором инфинитезимальной симметрии (или просто симметрией) разностного уравнения, если

$$
D_{Q}(K) \equiv 0
$$

где $D_{Q}-$ производная Фреше от $Q$, которая определяется как

$$
D_{Q}=\sum_{i, j} Q_{u_{i, j}} \mathcal{S}^{i} \mathcal{T}^{j}, \quad Q_{u_{i, j}}=\frac{\partial Q}{\partial u_{i, j}} .
$$

Для заданного $Q$ сумма в уравнении (2) является конечной. В случае квад-уравнения она содержит только четыре члена. Здесь производная $D_{Q}(K)$ равна нулю как элемент из $\mathcal{F}_{Q}$, т. е. $\mathcal{E}\left(D_{Q}(K)\right)=0$.

Если $K$ - симметрия, а $u=u(n, m)$ - решение разностного уравнения (2), то инфинитезимальное преобразование решения $u, \hat{u}=u+\epsilon K$, удовлетворяет уравнению

$$
Q\left(\hat{u}_{0,0}, \hat{u}_{1,0}, \hat{u}_{0,1}, \hat{u}_{1,1}\right) \equiv \mathcal{O}\left(\epsilon^{2}\right) .
$$

Если разностное уравнение $Q=0$ допускает симметрии, они образуют алгебру Ли. Действительно, с симметрией $K \in \mathcal{F}_{Q}$ можно связать эволюционное дифференцирование поля $\mathcal{F}_{Q}$ (или векторное поле на $\mathcal{F}_{Q}$ ):

$$
X_{K}=\sum_{(p, q) \in \mathbb{Z}^{2}} K_{p, q} \frac{\partial}{\partial u_{p, q}}
$$

где $K_{p, q}=\mathcal{S}^{p} \mathcal{T}^{q}(K)$. Дифференцирование (векторное поле) $X_{K}$ называется эволюционным, если оно коммутирует с автоморфизмами сдвигов: $\mathcal{S} X_{K}=X_{K} \mathcal{S}, \mathcal{T} X_{K}=$ $X_{K} \mathcal{T}$. Из условия (8) следует, что для любого $a \in J_{Q}$ имеем $\mathcal{E}\left(X_{K}(a)\right)=0$, и, таким образом, эволюционное дифференцирование $X_{K}$ корректно определено на разностном поле отношений $\mathcal{F}_{Q}$.

Если разностное уравнение допускает две симметрии $F$ и $G$, то коммутатор соответствующих эволюционных дифференцирований является эволюционным дифференцированием поля $\mathcal{F}_{Q}$ :

$$
X_{F} X_{G}-X_{G} X_{F}=X_{H},
$$

где $H=[F, G]$, а $[F, G]$ обозначает скобку Ли:

$$
[F, G]=X_{F}(G)-X_{G}(F)=D_{G}(F)-D_{F}(G) \in \mathcal{F}_{Q} .
$$

Нетрудно видеть, что $H$ является симметрией. Действительно,

$$
D_{Q}(H)=X_{H}(Q)=X_{F} X_{G}(Q)-X_{G} X_{F}(Q)=X_{F} D_{Q}(G)-X_{G} D_{Q}(F) \equiv 0 .
$$

Симметрии образуют линейное пространство над полем $\mathbb{C}$. Скобка (9) линейна по каждому аргументу, кососимметрична и удовлетворяет тождеству Якоби. Алгебру Ли симметрий разностного уравнения $Q=0$ будем обозначать через $\mathfrak{A}_{Q}$. Существование бесконечномерной алгебры Ли $\mathfrak{A}_{Q}$ представляет собой характеристическое свойство интегрируемых уравнений и может использоваться как определение интегрируемости. 
Подчеркнем, что симметрия $K$ является элементом поля $\mathcal{F}_{Q}$, поэтому она представима функцией $\mathcal{E}(K) \in \mathcal{F}_{0}$ переменных $U_{0}$. Как элемент поля $\mathcal{F}_{0}$ симметрия $K$ зависит от конечного набора переменных $\left\{u_{n, 0} \mid N_{1} \leqslant n \leqslant N_{2}\right\}$ и $\left\{u_{0, m} \mid M_{1} \leqslant n \leqslant M_{2}\right\}$, поэтому ее можно охарактеризовать четырьмя целыми числами $\left(N_{1}, N_{2}, M_{1}, M_{2}\right)$. Можно показать, что если

$$
K=K\left(u_{N_{1}, 0}, \ldots, u_{N_{2}, 0}, u_{0, M_{1}}, \ldots, u_{0, M_{2}}\right)
$$

является симметрией уравнения четырехугольников, то она представляет собой сумму двух функциий:

$$
K=K_{\mathbf{s}}\left(u_{N_{1}, 0}, \ldots, u_{N_{2}, 0}\right)+K_{\mathbf{t}}\left(u_{0, M_{1}}, \ldots, u_{0, M_{2}}\right) .
$$

В частности, для уравнений АБС известно [22], [29], что любая "пятиточечная симметрия" $K=K\left(u_{0,0}, u_{-1,0}, u_{0,-1}, u_{1,0}, u_{0,1}\right)$ представляет собой сумму двух симметрий: $K=K_{\mathbf{s}}\left(u_{-1,0}, u_{0,0}, u_{1,0}\right)+K_{\mathbf{t}}\left(u_{0,-1}, u_{0,0}, u_{0,1}\right)$. Таким образом, задача описания симметрии, по всей видимости, сводится к исследованию симметрий, зависящих от "одномерного" набора переменных $U_{\mathbf{s}}$ или $U_{\mathbf{t}}$. Более того, все уравнения АБС и уравнение Виале обладают бесконечной иерархией таких симметрий.

Пусть $f=f\left(u_{N_{1}, 0}, \ldots, u_{N_{2}, 0}\right) \in \mathcal{F}_{\mathbf{s}}$ и $\partial f / \partial u_{N_{1}, 0} \neq 0, \partial f / \partial u_{N_{2}, 0} \neq 0$. Тогда порядок функции $f$ определяется как $\operatorname{ord}_{\mathbf{s}}(f)=\left(N_{1}, N_{2}\right)$. Например,

$$
K^{(1)}=\left(u_{1,0}-u_{-1,0}\right)^{-1}
$$

является генератором симметрии для уравнения $(7)$ и $\operatorname{ord}_{\mathbf{s}}\left(K^{(1)}\right)=(-1,1)$. Аналогично, порядок некоторой функции $g=g\left(u_{0, M_{1}}, \ldots, u_{0, M_{2}}\right) \in \mathcal{F}_{\mathbf{t}}$ определяется как $\operatorname{ord}_{\mathbf{t}}(g)=\left(M_{1}, M_{2}\right)$.

ОПРЕДЕЛЕНИЕ 3. 1. Пара функций $\rho, \sigma \in \mathcal{F}_{Q}$ называется законом сохранения для разностного уравнения (2), если

$$
(\mathcal{T}-\mathbf{1})(\rho) \equiv(\mathcal{S}-\mathbf{1})(\sigma) .
$$

Функции $\rho$ и $\sigma$ будем называть плотностью и потоком закона сохранения, а 1 обозначает тождественное отображение.

2. Закон сохранения называется тривиальнъм, если функции $\rho$ и $\sigma$ являются компонентами (разностного) градиента некоторого элемента $H \in \mathcal{F}_{Q}$, т. е.

$$
\rho=(\mathcal{S}-\mathbf{1})(H), \quad \sigma=(\mathcal{T}-\mathbf{1})(H) .
$$

3. Непостоянный элемент $C \in \mathcal{F}_{Q}$ называется s-константой (соответственно, $\mathbf{t}$-константой), если $\mathcal{S}(C)=C$ (соответственно, $\mathcal{T}(C)=C$ ). Элементы из $\mathcal{F}_{Q}$, инвариантные относительно обоих сдвигов, называются константами поля $\mathcal{F}_{Q}$.

Существование нетривиальных законов сохранения и/или $\mathbf{s}-$ или t-констант для разностного уравнения является весьма редким свойством. У уравнений АБС (и многих других интегрируемых разностных уравнений) бесконечно много нетривиальных законов сохранения. Как и в дифференциальном случае [30], существование s- или t-констант указывает на то, что разностное уравнение является линеаризуемым. В качестве примера разностного уравнения с $\mathbf{s}-$ и $\mathbf{t}$-константами можно взять линейное уравнение с $Q=u_{0,0}-u_{1,0}-u_{0,1}+u_{1,1}$, которое имеет $\mathbf{t}$-константу $f\left(u_{1,0}-u_{0,0}\right)$ и $\mathbf{s}-$ константу $g\left(u_{0,1}-u_{0,0}\right)$, где $f, g-$ произвольные функции. 
ОПРЕДЕЛЕНИЕ 4. Пусть $\operatorname{Im}(\mathcal{S}-\mathbf{1})$ и $\operatorname{Im}(\mathcal{T}-\mathbf{1})$ - образы отображений $\mathcal{S}-\mathbf{1}: \mathcal{F}_{Q} \mapsto$ $\mathcal{F}_{Q}$ и $\mathcal{T}-1: \mathcal{F}_{Q} \mapsto \mathcal{F}_{Q}$.

1. Два элемента $\rho, \varrho \in \mathcal{F}_{Q}$ являются $\mathbf{s - э к в и в а л е н т н ы м и ~}\left(\rho \cong_{\mathbf{s}} \varrho\right)$, если $\rho-\varrho \in$ $\operatorname{Im}(\mathcal{S}-\mathbf{1})$. Элементы факторпространства $\mathcal{F}_{Q} / \operatorname{Im}(\mathcal{S}-\mathbf{1})$ будем называть s-функиионалами, или плотностями.

2. Два элемента $\sigma, \varsigma \in \mathcal{F}_{Q}$ являются $\mathbf{t}$-эквивалентными $\left(\sigma \cong_{\mathbf{t}} \varsigma\right)$, если $\sigma-\varsigma \in$ $\operatorname{Im}(\mathcal{T}-\mathbf{1})$. Элементы факторпространства $\mathcal{F}_{Q} / \operatorname{Im}(\mathcal{T}-\mathbf{1})$ будем называть $\mathbf{t}$-функиионалами, или потоками.

В общем случае проверка $\mathbf{s}-$ или $\mathbf{t}$-эквивалентности является весьма сложной задачей. Эта задача имеет простое решение в том случае, когда плотности являются элементами $\mathcal{F}_{\mathbf{s}}$, или в случае, когда потоки принадлежат $\mathcal{F}_{\mathbf{t}}$. В разделе 4 мы покажем, что интегрируемое квад-уравнение (такое, как уравнение Виале) обладает бесконечной иерархией канонических законов сохранения таких, что их плотности являются элементами из $\mathcal{F}_{\mathbf{s}}$.

Для элементов из поля $\mathcal{F}_{\mathbf{s}}$ имеется критерий, позволяющий определить, являются ли они s-эквивалентными. Он основан на понятии вариационной производной (или на операторе Эйлера).

ОПРЕДЕЛЕНИЕ 5. Пусть $f \in \mathcal{F}_{\mathbf{s}}$ имеет порядок $\left(N_{1}, N_{2}\right)$. Тогда вариационная производная $\delta_{\mathbf{s}}$ элемента $f$ определяется как

$$
\delta_{\mathbf{s}}(f)=\sum_{k=N_{1}}^{N_{2}} \mathcal{S}^{-k}\left(\frac{\partial f}{\partial u_{k, 0}}\right) .
$$

Если два элемента $\rho, \varrho \in \mathcal{F}_{\mathbf{s}}$ эквивалентны, $\rho \cong_{\mathbf{s}} \varrho$, то $\delta_{\mathbf{s}}(\rho)=\delta_{\mathbf{s}}(\varrho)$. В частности, если плотность $\rho \in \mathcal{F}_{\mathbf{s}}$ тривиальна, то $\delta_{\mathbf{s}}(\rho)=0$. Порядок плотности $\rho \in \mathcal{F}_{\mathbf{s}}$ определяется как $\operatorname{ord}_{\mathbf{s}}(\rho)=N_{2}-N_{1}$, где $\left(N_{1}, N_{2}\right)=\operatorname{ord}\left(\delta_{\mathbf{s}}(\rho)\right)$. Эквивалентные плотности имеют одинаковый порядок. Например, плотности законов сохранения для уравнения $\mathrm{H1}$ (7)

$$
\rho_{0}=\ln \left(u_{1,0}-u_{-1,0}\right), \quad \rho_{1}=\frac{1}{\left(u_{1,0}-u_{-1,0}\right)\left(u_{2,0}-u_{0,0}\right)}
$$

имеют порядки 4 и 6 соответственно.

\section{3. РЕКУРСИОННЫЙ ОПЕРАТОР И КАНОНИЧЕСКИЕ ЗАКОНЫ СОХРАНЕНИЯ}

В данном разделе мы даем определения (s- и t-) разностных и псевдоразностных операторов. Мы адаптируем концепцию рекурсионных операторов к разностным уравнениям и определяем критерии того, является ли псевдоразностный оператор рекурсионным оператором разностного квад-уравнения. Используя формальные ряды Лорана и их вычеты, можно вывести бесконечно много условий интегрируемости для разностных квад-уравнений.

3.1. Рекурсионные операторы разностных уравнений. Определим s- и t-псевдоразностные операторы.

ОПРЕДЕЛЕНИЕ 6. s-Разностный оператор $B$ порядка $N$ с коэффициентами из $\mathcal{F}_{Q}$ представляет собой конечную сумму вида

$$
B=b_{N} \mathcal{S}^{N}+b_{N-1} \mathcal{S}^{N-1}+\cdots+b_{M} \mathcal{S}^{M}, \quad b_{k} \in \mathcal{F}_{Q}, \quad M \leqslant N, \quad N, M \in \mathbb{Z} .
$$




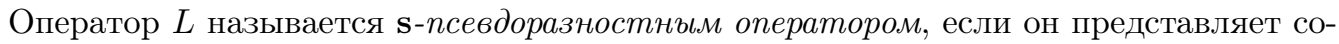
бой или конечную сумму слагаемых

$$
L=\sum B_{n} \circ C_{n}^{-1}
$$

где $B_{n}$ и $C_{n}$-s-разностные операторы с коэффициентами из $\mathcal{F}_{Q}$, а о обозначает их композицию, или конечную композицию s-псевдоразностных операторов вида (14).

В приведенном выше определении мы использовали отображение сдвига $\mathcal{S}$ и таким образом определили s-разностный и s-псевдоразностный операторы. Используя отображение сдвига $\mathcal{T}$, можно определить $\mathbf{t}$-разностный и $\mathbf{t}$-псевдоразностный операторы. Далее мы будем опускать s- (или t-) и говорить просто о разностном или псевдоразностном операторах, если это ясно из контекста.

Имеется естественное действие разностных операторов на элементы поля $\mathcal{F}_{Q}$, однако действие псевдоразностных операторов, как правило, не определено. Действие псевдоразностного оператора $L(14)$, где $B_{n}$ и $C_{n}$-s-разностные операторы, можно определить на тех элементах из $a_{i} \in \mathcal{F}_{Q}$, которые принадлежат пересечению пространств образов разностных операторов $C_{n}$, так что действие операторов $C_{n}^{-1}\left(a_{i}\right)$ определено, т. е. $C_{n}^{-1}\left(a_{i}\right) \in \mathcal{F}_{Q}$. Линейное пространство, являющееся $\mathbb{C}$-линейной оболочкой всех таких элементов $a_{i}$, представляет собой область определения псевдоразностного оператора (14), обозначаемого как $\operatorname{Dom}(L)$.

Под рекурсионным оператором $\mathfrak{R}$ разностного уравнения $Q=0$ мы будем понимать такой s-псевдоразностный оператор, что

$$
\mathfrak{R}: \operatorname{Dom}(\mathfrak{R}) \cap \mathfrak{A}_{Q} \mapsto \mathfrak{A}_{Q},
$$

где $\mathfrak{A}_{Q}$ - линейное пространство симметрий данного разностного уравнения. Другими словами, если действие оператора $\mathfrak{R}$ на симметрию $K \in \mathcal{F}_{0}$ определено, т. е. $\mathfrak{R}(K) \in \mathcal{F}_{Q}$, то $\mathfrak{R}(K)$ представляет собой симметрию того же разностного уравнения. Аналогично определяется $\mathbf{t}$-псевдоразностный рекурсионный оператор $\widehat{\Re}: \operatorname{Dom}(\widehat{\Re}) \cap$ $\mathfrak{A}_{Q} \mapsto \mathfrak{A}_{Q}$. Оператор умножения на постоянную является тривиальным рекурсионным оператором. Ниже мы будем предполагать, что операторы $\mathfrak{R}$ и $\widehat{\Re}$ нетривиальны.

Tеорема 1. Пусть $Q\left(u_{0,0}, u_{1,0}, u_{0,1}, u_{1,1}\right)=0$ - разностное уравнение. Тогда справедливы следующие утверждения.

1. Если существуют два $\mathbf{s}-$ псевдоразностных оператора $\mathfrak{R}$ и $\mathfrak{P}$ такие, что

$$
D_{Q} \circ \mathfrak{R}=\mathfrak{P} \circ D_{Q}
$$

то $\Re$ является рекурсионным оператором разностного уравнения.

2. Соотношение (15) справедливо тогда и только тогда, когда

$$
\mathcal{T}(\mathfrak{R})-\mathfrak{R}=\left[\Phi \circ \Re, \Phi^{-1}\right]
$$

где $\Phi=\left(Q_{u_{1,1}} \mathcal{S}+Q_{u_{0,1}}\right)^{-1} \circ\left(Q_{u_{1,0}} \mathcal{S}+Q_{u_{0,0}}\right)$ и оператор $\mathfrak{P}$ удовлетворяет соотношению

$$
\mathfrak{P}=\left(Q_{u_{1,0}} \mathcal{S}+Q_{u_{0,0}}\right) \circ \mathfrak{R} \circ\left(Q_{u_{1,0}} \mathcal{S}+Q_{u_{0,0}}\right)^{-1}
$$


ДокАЗАтЕЛЬство. 1. Если $K$ является симметрией и $\mathfrak{R}(K) \in \mathcal{F}_{Q}$, то из (15) следует, что $D_{Q} \mathfrak{R}(K)=\mathfrak{P} D_{Q}(K) \equiv 0$, и, таким образом, $\mathfrak{R}(K)$ является симметрией.

2. Представим $D_{Q}$ в факторизованном виде:

$$
D_{Q}=Q_{u_{1,1}} \mathcal{S} \circ \mathcal{T}+Q_{u_{0,1}} \mathcal{T}+Q_{u_{1,0}} \mathcal{S}+Q_{u_{0,0}}=\left(Q_{u_{1,1}} \mathcal{S}+Q_{u_{0,1}}\right) \circ(\mathcal{T}+\Phi),
$$

где

$$
\Phi=\left(Q_{u_{1,1}} \mathcal{S}+Q_{u_{0,1}}\right)^{-1} \circ\left(Q_{u_{1,0}} \mathcal{S}+Q_{u_{0,0}}\right) .
$$

Тогда соотношение (15) можно переписать как

$$
\mathcal{T}(\mathfrak{R}) \circ \mathcal{T}+\Phi \circ \mathfrak{R}=\left(Q_{u_{1,1}} \mathcal{S}+Q_{u_{0,1}}\right)^{-1} \circ \mathfrak{P} \circ\left(Q_{u_{1,1}} \mathcal{S}+Q_{u_{0,1}}\right) \circ(\mathcal{T}+\Phi),
$$

что эквивалентно

$$
\mathcal{T}(\mathfrak{R})=\left(Q_{u_{1,1}} \mathcal{S}+Q_{u_{0,1}}\right)^{-1} \circ \mathfrak{P} \circ\left(Q_{u_{1,1}} \mathcal{S}+Q_{u_{0,1}}\right)
$$

и

$$
\Phi \circ \mathfrak{R}=\left(Q_{u_{1,1}} \mathcal{S}+Q_{u_{0,1}}\right)^{-1} \circ \mathfrak{P} \circ\left(Q_{u_{1,1}} \mathcal{S}+Q_{u_{0,1}}\right) \circ \Phi .
$$

Используя (18), можно переписать (21) в виде (17). Из (20) и (21) следует, что

$$
\Phi \circ \Re=\mathcal{T}(\mathfrak{R}) \circ \Phi
$$

Последнее равенство можно переписать в виде (16).

Аналогично можно доказать похожее утверждение для $\mathcal{T}$-направления.

Tеорема 2. Пусть $Q\left(u_{0,0}, u_{1,0}, u_{0,1}, u_{1,1}\right)=0$ - разностное уравнение. Тогда справедливы следующие утверждения.

1. Если существуют два $\mathbf{t}-$ псевдоразностных оператора $\widehat{\mathfrak{R}}$ и $\widehat{\mathfrak{P}}$ такие, что

$$
D_{Q} \circ \widehat{\mathfrak{R}}=\widehat{\mathfrak{P}} \circ D_{Q},
$$

то $\widehat{\Re}$ является рекурсионным оператором разностного уравнения.

2. Соотношение (23) справедливо тогда и только тогда, когда

$$
\mathcal{S}(\widehat{\mathfrak{R}})-\widehat{\mathfrak{R}}=\left[\Psi \circ \widehat{\mathfrak{R}}, \Psi^{-1}\right],
$$

где $\Psi=\left(Q_{u_{1,1}} \mathcal{T}+Q_{u_{1,0}}\right)^{-1} \circ\left(Q_{u_{0,1}} \mathcal{T}+Q_{u_{0,0}}\right)$ и оператор $\widehat{\mathfrak{P}}$ можно записать как

$$
\widehat{\mathfrak{P}}=\left(Q_{u_{0,1}} \mathcal{T}+Q_{u_{0,0}}\right) \circ \widehat{\mathfrak{R}} \circ\left(Q_{u_{0,1}} \mathcal{T}+Q_{u_{0,0}}\right)^{-1} \text {. }
$$

Как и в случае дифференциальных уравнений в частных производных, в следующем утверждении мы покажем, что если $\mathfrak{R}$ является рекурсионным оператором, то и $\mathfrak{R}^{n}$ также является рекурсионным оператором для всех $n \in \mathbb{Z}$.

СлЕДСТвиЕ. 1. Если выполняются условия 1 из теоремы 1, то псевдоразностный оператор $\Re$ удовлетворяет уравнениям

$$
\mathcal{T}\left(\mathfrak{R}^{n}\right)-\mathfrak{R}^{n}=\left[\Phi \circ \mathfrak{R}^{n}, \Phi^{-1}\right], \quad n \in \mathbb{Z} .
$$

2. Если выполняются условия 1 из теоремы 2, то псевдоразностный оператор $\widehat{\Re}$ удовлетворяет уравнениям

$$
\mathcal{S}\left(\widehat{\mathfrak{R}}^{n}\right)-\widehat{\mathfrak{R}}^{n}=\left[\Psi \circ \widehat{\mathfrak{R}}^{n}, \Psi^{-1}\right] \quad n \in \mathbb{Z} .
$$


ДокАЗАТЕЛЬство. Из соотношения (15) вытекает, что $D_{Q} \circ \mathfrak{R}^{n}=\mathfrak{P}^{n} \circ D_{Q}, n \in \mathbb{Z}$. Таким образом, можно применить теорему 1 к $\mathfrak{R}^{n}$ и $\mathfrak{P}^{n}$ и получить (25) из (16). Доказательство второго утверждения (26) следствия аналогично.

Во всех наших определениях и утверждениях имеется очевидная симметрия между $\mathcal{T}$ - и $\mathcal{S}$-объектами (это относится к симметриям, псевдоразностным операторам, симплектическим и гамильтоновым операторам). Ниже мы сосредоточимся на $\mathcal{S}$-объектах, однако, если это нам потребуется, будем формулировать некоторые утверждения для обоих случаев.

\section{2. Канонические законы сохранения и условия интегрируемости.}

ОпрЕдЕЛЕниЕ 7. Формалъные рядъ (Лорана ${ }^{1)}$ ) порядка $N$ определяются как формальная полубесконечная сумма

$$
A=a_{N} \mathcal{S}^{N}+\cdots+a_{0}+a_{-1} \mathcal{S}^{-1}+\cdots, \quad a_{N} \neq 0, \quad a_{k} \in \mathcal{F}_{Q}, \quad N \in \mathbb{Z} .
$$

Формальные ряды Лорана образуют тело. Суммы и произведения (композиции) формальных рядов представляют собой формальные ряды. Произведение является ассоциативным, но не коммутативным. Для любого формального ряда $A$ существует формальный ряд $A^{-1}$ такой, что $A \circ A^{-1}=A^{-1} \circ A=1$. Чтобы найти первые $n$ коэффициентов ряда $A^{-1}$, необходимо знать в точности первые $n$ коэффициентов ряда $A$. То же верно и для композиции двух формальных рядов. Например, для двух формальных рядов Лорана - ряда $A$, определенного с помощью $(27)$, и ряда $B=b_{M} \mathcal{S}^{M}+b_{M-1} \mathcal{S}^{M-1}+\cdots-$ имеем

$$
A \circ B=\sum_{k=0}^{\infty} c_{N+M-k} \mathcal{S}^{N+M-k}, \quad c_{k}=\sum_{q=0}^{N+M-k} a_{N-q} \mathcal{S}^{N-q}\left(b_{q+k-N}\right) \in \mathcal{F}_{Q} .
$$

Любой псевдоразностный оператор $B$ можно единственным образом представить с помощью формального ряда Лорана $B_{\mathrm{L}}$. Например, для $B=(a \mathcal{S}+b)^{-1}$ имеем

$$
B_{\mathrm{L}}=\alpha_{-1} \mathcal{S}^{-1}+\alpha_{-2} \mathcal{S}^{-2}+\alpha_{-3} \mathcal{S}^{-3}+\cdots,
$$

где коэффициенты $\alpha_{k} \in \mathcal{F}_{Q}$ можно найти по рекуррентной формуле:

$$
\alpha_{-1}=\mathcal{S}^{-1}\left(\frac{1}{a}\right), \quad \alpha_{-n}=-\mathcal{S}^{-1}\left(\frac{\alpha_{1-n} b}{a}\right) .
$$

ОПредЕЛЕНиЕ 8. Пусть $A_{\mathrm{L}}$ обозначает представление псевдоразностного оператора $A$ в виде ряда Лорана. Тогда ord $A_{\mathrm{L}}$ называется лорановым порядком оператоpa $A$.

1) Можно также определить формальный ряд Тейлора порядка $-N$ как полубесконечную сумму

$$
C=c_{-N} \mathcal{S}^{-N}+c_{1-N} \mathcal{S}^{1-N}+\cdots+c_{-1} \mathcal{S}^{-1}+c_{0}+c_{1} \mathcal{S}+\cdots, \quad c_{N} \neq 0, \quad c_{k} \in \mathcal{F}_{Q}, \quad N \in \mathbb{Z}
$$

и развить аналогичную теорию, основанную на ряде Тейлора (или на обоих рядах). Однако в случае, когда рекурсионный оператор представляет собой композицию гамильтоновых и симплектических операторов, можно показать, что оба подхода дают эквивалентные результаты. Таким образом, в применении к уравнениям АБС и уравнению Виале мы ограничимся формальными рядами Лорана. Исследование обоих рядов (и Лорана, и Тейлора) имеет смысл для асимметричных разностных уравнений. 
Теперь введем вычет формального ряда, а также разностную версию теоремы Адлера [31], которая будет использоваться для получения условий интегрируемости для разностных квад-уравнений.

ОПРЕДЕЛЕНИЕ 9. Пусть $A$ является формальным рядом порядка $N$ (см. (27)). Вычет $\operatorname{res} A$ и логарифмический вычет $\operatorname{res} \ln A$ определяются как

$$
\operatorname{res} A=a_{0}, \quad \operatorname{res} \ln A=\ln a_{N} .
$$

Нам потребуется следующий разностный аналог теоремы Адлера [31].

TEOPEMA 3. Пусть $A=a_{N} \mathcal{S}^{N}+a_{N-1} \mathcal{S}^{N-1}+\cdots u B=b_{M} \mathcal{S}^{M}+b_{M-1} \mathcal{S}^{M-1}+\cdots$ - два формалъных ряда Лорана порядков $N$ и $M$. Тогда

$$
\operatorname{res}[A, B]=(\mathcal{S}-\mathbf{1})(\sigma(A, B))
$$

где

$$
\sigma(A, B)=\sum_{n=1}^{N} \sum_{k=1}^{n} \mathcal{S}^{-k}\left(a_{-n}\right) \mathcal{S}^{n-k}\left(b_{n}\right)-\sum_{n=1}^{M} \sum_{k=1}^{n} \mathcal{S}^{-k}\left(b_{-n}\right) \mathcal{S}^{n-k}\left(a_{n}\right) \in \mathcal{F}_{Q} .
$$

ДокАЗАТЕЛЬСТво. Вклад в вычет дают только коммутаторы вида $\left[a_{p} \mathcal{S}^{p}\right.$, $\left.b_{-p} \mathcal{S}^{-p}\right], p \in \mathbb{Z}$. Предполагая $p>0$, получаем

$$
\begin{aligned}
{\left[a_{p} \mathcal{S}^{p}, b_{-p} \mathcal{S}^{-p}\right] } & =a_{p} \mathcal{S}^{p}\left(b_{-p}\right)-\mathcal{S}^{-p}\left(a_{p}\right) b_{-p}=\left(\mathcal{S}^{p}-\mathbf{1}\right)\left(\mathcal{S}^{-p}\left(a_{p}\right) b_{-p}\right)= \\
& =(\mathcal{S}-\mathbf{1}) \sum_{k=1}^{p} \mathcal{S}^{p-k}\left(\mathcal{S}^{-p}\left(a_{p}\right) b_{-p}\right) .
\end{aligned}
$$

Для формального ряда $A$ первого порядка первые $n$ коэффициентов ряда полностью определяют конечную последовательность первых $n$ вычетов $\rho_{0}=\operatorname{res} \ln A$, $\rho_{1}=\operatorname{res} A, \ldots, \rho^{n-1}=\operatorname{res} A^{n-1}$.

В теориии псевдодифференциальных формальных рядов (см., например, [4], [6], [7]) для ряда $A=a D_{x}^{N}+b D_{x}^{N-1}+\cdots$ порядка $N$ всегда можно найти $N$-й корень, т. е. формальный ряд

$$
B=A^{1 / N}=a^{1 / N} D_{x}+\left(\frac{1}{N} a^{(1 / N)-1} b-\frac{N-1}{N} a^{(1 / N)-1} D_{x}(a)\right)+\cdots
$$

такой, что $B^{N}=A$, и, следовательно, параметризовать коэффициенты ряда $A$ последовательностью канонических вычетов $\operatorname{res} \ln B, \operatorname{res} B, \operatorname{res} B^{2}, \ldots$ Вообще говоря, в случае формального разностного ряда невозможно найти $N$-й корень ряда $A=a_{N} \mathcal{S}^{N}+a_{N-1} \mathcal{S}^{N-1}+\cdots$, т. е. ряд первого порядка $B=\alpha_{1} \mathcal{S}+\alpha_{0}+\cdots$ с коэффициентами в $\mathcal{F}_{Q}$ такой, что $B^{N}=A$. Действительно, вычисления показывают, что для нахождения коэффициента $\alpha_{k} \in \mathcal{F}_{Q}$ необходимо решить уравнения вида

$$
\prod_{i=0}^{N-1} \mathcal{S}^{i}\left(\alpha_{1}\right)=a_{N}, \quad \alpha_{k}+\mathcal{S}\left(\alpha_{k}\right)+\cdots+\mathcal{S}^{N-1}\left(\alpha_{k}\right)=g_{k}, \quad k=0,-1,-2, \ldots,
$$

для некоторых $g_{k} \in \mathcal{F}_{Q}$. Очевидно, решение $\alpha_{k} \in \mathcal{F}_{Q}$ не существует, если $g_{k} \notin$ $\operatorname{Im}\left(\mathbf{1}+\mathcal{S}+\cdots+\mathcal{S}^{N-1}\right)$. 
ТЕОрема 4. Если разностное уравнение обладает рекурсионным оператором $\Re$, $\operatorname{ord}_{\mathrm{L}}(\mathfrak{R})=N>0$, то оно имеет бесконечно много канонических законов сохранения

$$
(\mathcal{T}-\mathbf{1}) \rho_{n N}=(\mathcal{S}-\mathbf{1}) \sigma_{n N}, \quad n=0,1,2, \ldots,
$$

с каноническими плотностями

$$
\rho_{0}=\operatorname{res} \ln \Re_{\mathrm{L}}, \quad \rho_{n N}=\operatorname{res} \mathfrak{R}_{\mathrm{L}}^{n}, \quad n>0 .
$$

ДокАзАтЕльство. Уравнение (25) очевидным образом удовлетворяется, если заменить $\Phi$ и $\mathfrak{R}$ на соответствующий формальный ряд Лорана $\Phi_{\mathrm{L}}$ и $\mathfrak{R}_{\mathrm{L}}$. Беря вычет уравнения (25) при $n \geqslant 1$, получаем

$$
\mathcal{T}\left(\operatorname{res} \Re_{\mathrm{L}}^{n}\right)-\operatorname{res} \Re_{\mathrm{L}}^{n}=\operatorname{res}\left(\left[\Phi_{\mathrm{L}} \circ \mathfrak{R}_{\mathrm{L}}^{n}, \Phi_{\mathrm{L}}^{-1}\right]\right)=(\mathcal{S}-\mathbf{1}) \sigma\left(\Phi_{\mathrm{L}} \circ \mathfrak{R}_{\mathrm{L}}^{n}, \Phi_{\mathrm{L}}^{-1}\right) .
$$

Из теоремы 3 следует, что

$$
\sigma_{n N}=\sigma\left(\Phi_{\mathrm{L}} \circ \Re_{\mathrm{L}}^{n}, \Phi_{\mathrm{L}}^{-1}\right), \quad \sigma_{n} \in \mathcal{F}_{Q} .
$$

При $n=0$ перепишем (22) в виде

$$
\Phi \circ \mathfrak{R} \circ \Phi^{-1} \circ \mathfrak{R}^{-1}=\mathcal{T}(\mathfrak{R}) \circ \mathfrak{R}^{-1}
$$

и возьмем его логарифмический вычет. Это приводит к равенству

$$
\mathcal{T}\left(\operatorname{res} \ln \Re_{\mathrm{L}}\right)-\operatorname{res} \ln \Re_{\mathrm{L}}=(\mathcal{S}-\mathbf{1}) \sum_{k=0}^{N-1} \mathcal{S}^{k-1}\left(\ln \alpha_{0}\right)
$$

где $\alpha_{0}-$ первый коэффициент в разложении $\Phi_{\mathrm{L}}=\alpha_{0}+\alpha_{1} \mathcal{S}^{-1}+\cdots($ см. (35)).

В теореме 4 номера плотностей и потоков вычисляются по модулю $N$, где $N-$ лорановский порядок рекурсионного оператора.

Если рекурсионный оператор известен, теорема 4 дает алгоритм для явного нахождения последовательности законов сохранения, включая как плотности $\rho_{k}$, так и соответствующие потоки $\sigma_{k}$. Вычеты степеней формального ряда несложно вычислить. Например, если

$$
\mathfrak{R}=r_{1} \mathcal{S}+r_{0}+r_{-1} \mathcal{S}^{-1}+r_{-2} \mathcal{S}^{-2}+r_{-3} \mathcal{S}^{-3}+\cdots
$$

Tо

$$
\begin{aligned}
\operatorname{res} \ln \Re= & \ln r_{1}, \quad \operatorname{res} \Re=r_{0}, \quad \operatorname{res} \Re^{2}=\mathcal{S}^{-1}\left(r_{1}\right) r_{-1}+r_{0}^{2}+r_{1} \mathcal{S}^{-1}\left(r_{-1}\right), \\
\operatorname{res} \mathfrak{R}^{3}= & \mathcal{S}^{-2}\left(r_{1}\right) \mathcal{S}^{-1}\left(r_{1}\right) r_{-2}+\mathcal{S}^{-1}\left(r_{0}\right) \mathcal{S}^{-1}\left(r_{1}\right) r_{-1}+2 \mathcal{S}^{-1}\left(r_{1}\right) r_{-1} r_{0}+ \\
& +\mathcal{S}^{-1}\left(r_{1}\right) r_{1} \mathcal{S}\left(r_{-2}\right)+r_{0}^{3}+2 r_{0} r_{1} \mathcal{S}\left(r_{-2}\right)+r_{1} \mathcal{S}\left(r_{-1}\right) \mathcal{S}\left(r_{0}\right)+ \\
& +r_{1} \mathcal{S}\left(r_{1}\right) \mathcal{S}^{2}\left(r_{-2}\right) .
\end{aligned}
$$

ПРЕДЛОЖЕНИЕ 1. Если рекурсионный оператор $\Re$ представляется формалъным рядом первого порядка, $\mathfrak{R}_{\mathrm{L}}=r_{1} \mathcal{S}+r_{0}+r_{-1} \mathcal{S}^{-1}+\cdots$, mо

$$
\begin{aligned}
(\mathcal{T}-\mathbf{1})\left(\ln r_{1}\right) & =(\mathcal{S}-\mathbf{1}) \mathcal{S}^{-1}\left(\ln \frac{Q_{u_{1,1}}}{Q_{u_{1,0}}}\right), \\
(\mathcal{T}-\mathbf{1})\left(r_{0}\right) & =(\mathcal{S}-\mathbf{1}) \mathcal{S}^{-1}\left(r_{1} F\right), \\
(\mathcal{T}-\mathbf{1})\left(r_{-1} \mathcal{S}^{-1}\left(r_{1}\right)+r_{0}^{2}+r_{1} \mathcal{S}\left(r_{-1}\right)\right) & =(\mathcal{S}-\mathbf{1})\left(\sigma_{2}\right),
\end{aligned}
$$


əəe

$$
\sigma_{2}=\mathcal{S}^{-1}\left(r_{1} F\right)\left\{\mathcal{S}^{-1}\left(r_{0}\right)+r_{0}-\mathcal{S}^{-2}\left(r_{1} F\right)\right\}-\left(1+\mathcal{S}^{-1}\right)\left(r_{1} G \mathcal{S}^{-1}\left(r_{1} F\right)\right)
$$

и мы использовали обозначения

$$
F=\frac{Q_{u_{0,1}} \mathcal{S}^{-1}\left(Q_{u_{1,0}}\right)-Q_{u_{0,0}} \mathcal{S}^{-1}\left(Q_{u_{1,1}}\right)}{Q_{u_{1,0}} \mathcal{S}^{-1}\left(Q_{u_{1,1}}\right)}, \quad G=\frac{Q_{u_{0,0}}}{Q_{u_{1,0}}} .
$$

ДокаЗАтеЛьСтво. Разложим $\Phi$ и $\Phi^{-1}(18)$ в формальный ряд Лорана:

$$
\Phi_{\mathrm{L}}=\alpha_{0}+\alpha_{1} \mathcal{S}^{-1}+\alpha_{2} \mathcal{S}^{-2}+\cdots, \quad \Phi_{\mathrm{L}}^{-1}=\beta_{0}+\beta_{1} \mathcal{S}^{-1}+\beta_{2} \mathcal{S}^{-2}+\cdots
$$

Коэффициенты $\alpha_{k}, \beta_{k} \in \mathcal{F}_{Q}$ можно найти по рекуррентным формулам:

$$
\begin{aligned}
\alpha_{0} & =\mathcal{S}^{-1}\left(\frac{Q_{u_{1,0}}}{Q_{u_{1,1}}}\right), \\
\alpha_{1} & =\mathcal{S}^{-1}\left(\frac{Q_{u_{0,0}}}{Q_{u_{1,1}}}-\frac{Q_{u_{0,1}}}{Q_{u_{1,1}}} \mathcal{S}^{-1}\left(\frac{Q_{u_{1,0}}}{Q_{u_{1,1}}}\right)\right), \\
\alpha_{n+1} & =(-1)^{n} \mathcal{S}^{-1}\left(\frac{Q_{u_{0,1}}}{Q_{u_{1,1}}} \alpha_{n}\right), \quad n \geqslant 1, \\
\beta_{0} & =\mathcal{S}^{-1}\left(\frac{Q_{u_{1,1}}}{Q_{u_{1,0}}}\right), \\
\beta_{1} & =\mathcal{S}^{-1}\left(\frac{Q_{u_{0,1}}}{Q_{u_{1,0}}}-\frac{Q_{u_{0,0}}}{Q_{u_{1,0}}} \mathcal{S}^{-1}\left(\frac{Q_{u_{1,1}}}{Q_{u_{1,0}}}\right)\right), \\
\beta_{n+1} & =(-1)^{n} \mathcal{S}^{-1}\left(\frac{Q_{u_{0,0}}}{Q_{u_{1,0}}} \beta_{n}\right), \quad n \geqslant 1 .
\end{aligned}
$$

Тогда соотношение (32) следует из (30), а формулы (33) и (34) следуют из (28) и (29) при $n=1$ и $n=2$ соответственно.

Можно провести вычисления для уравнений, соответствующих $n=3,4,5$ и т. д. Конечно, выражения становятся более громоздкими. Очевидно, что если мы рассмотрим рекурсионные операторы $\widehat{\mathfrak{R}}$ в $\mathcal{T}$-направлении, то мы немедленно получим

ПрЕДЛОЖЕНИЕ 2. Если рекурсионный оператор $\widehat{\Re}$ представляется формалъным $\mathcal{T}$-рядом Лорана первого порядка $\widehat{\Re}_{\mathrm{L}}=\hat{r}_{1} \mathcal{T}+\hat{r}_{0}+\hat{r}_{-1} \mathcal{T}^{-1}+\cdots$, mо

$$
\begin{aligned}
(\mathcal{S}-\mathbf{1})\left(\ln \hat{r}_{1}\right) & =(\mathcal{T}-\mathbf{1}) \mathcal{T}^{-1}\left(\ln \frac{Q_{u_{1,1}}}{Q_{u_{0,1}}}\right), \\
(\mathcal{S}-\mathbf{1})\left(\hat{r}_{0}\right) & =(\mathcal{T}-\mathbf{1}) \mathcal{T}^{-1}\left(\hat{r}_{1} \widehat{F}\right), \\
(\mathcal{S}-\mathbf{1})\left(\hat{r}_{-1} \mathcal{S}^{-1}\left(\hat{r}_{1}\right)+\hat{r}_{0}^{2}+\hat{r}_{1} \mathcal{S}\left(\hat{r}_{-1}\right)\right) & =(\mathcal{T}-\mathbf{1})\left(\hat{\sigma}_{2}\right),
\end{aligned}
$$

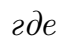

$$
\hat{\sigma}_{2}=\mathcal{S}^{-1}\left(\hat{r}_{1} \widehat{F}\right)\left\{\mathcal{S}^{-1}\left(\hat{r}_{0}\right)+\hat{r}_{0}-\mathcal{S}^{-2}\left(\hat{r}_{1} \widehat{F}\right)\right\}-\left(1+\mathcal{S}^{-1}\right)\left(\hat{r}_{1} \widehat{G} \mathcal{S}^{-1}\left(\hat{r}_{1} \widehat{F}\right)\right)
$$


и мы использовали обозначения

$$
\widehat{F}=\frac{Q_{u_{1,0}} \mathcal{T}^{-1}\left(Q_{u_{0,1}}\right)-Q_{u_{0,0}} \mathcal{T}^{-1}\left(Q_{u_{1,1}}\right)}{Q_{u_{0,1}} \mathcal{T}^{-1}\left(Q_{u_{1,1}}\right)}, \quad \widehat{G}=\frac{Q_{u_{0,0}}}{Q_{u_{0,1}}} .
$$

Можно показать, что существование формального ряда, удовлетворяющего уравнению (16), является необходимым условием существования иерархии высших симметрий (возрастающего порядка) для разностного уравнения. Таким образом, формулы (32)-(34) и (36)-(38) можно рассматривать как условия интегрируемости для разностного уравнения, определяемого функцией $Q$. Действительно, правые части в (32) и (36) выражаются только через производные от $Q$, и тот факт, что соответствующие левые части лежат в пространстве образов соответствующих операторов $\mathcal{T}-\mathbf{1}$ или $\mathcal{S}-\mathbf{1}$, является нетривиальным условием. Если это условие выполнено, то можно найти коэффициенты $r_{1}$ и $\hat{r}_{1}$ и перейти к следующим условиям $(33)$ и $(37)$ и т. д.

Теорема 4 позволяет произвести бесконечно много условий интегрируемости такого типа. Условия интегрируемости (32) и (36) были ранее получены Леви и Ямиловым [26] из анализа симметрий разностных уравнений. На самом деле они получили еще два условия этого типа, которые для нашего рассмотрения соответствуют формальному ряду Тейлора (см. сноску 1) и которые легко воспроизвести. Используя формальный ряд Тейлора, можно удвоить число условий, что подошло бы к случаю асимметричных или линеаризуемых уравнений.

Применение полученных условий интегрируемости не является простым упражнением. Тем не менее их можно прекрасно использовать как для анализа интегрируемости, так и для классификации интегрируемых разностных уравнений (см., например, [26]).

\section{4. РЕКУРСИОННЫЙ ОПЕРАТОР, СИММЕТРИИ И ЗАКОНЫ СОХРАНЕНИЯ ДЛЯ УРАВНЕНИЯ ВИАЛЕ}

Применим развитую в предыдущих разделах теорию к случаю уравнения Виале, а также к уравнениям АБС. Уравнение Виале характеризуется как интегрируемое, поскольку его алгебраическая энтропия обращается в нуль [21], в то время как интегрируемость уравнений АБС следует из их 3D-совместности [18]. Мы покажем, что эти уравнения являются интегрируемыми также в том смысле, что они допускают бесконечные иерархии симметрий, которые могут быть построены при помощи рекурсионных операторов. Чтобы сделать наше изложение замкнутым, мы сначала приведем некоторые свойства этих уравнений. Затем мы представим две симметрии и рекурсионный оператор для уравнения Виале и на явном примере продемонстрируем, как он работает в случае уравнений АБС. Наконец, мы приведем несколько первых членов бесконечной последовательности законов сохранения высших порядков для всех рассматриваемых уравнений.

Мы будем полагать, что многочлен $Q$ уравнения (1) является неприводимым. Это означает, что параметры $a_{i}$ не могут принимать значений, при которых $Q$ можно факторизовать в произведение двух многочленов. С помощью подходящего выбора коэффициентов $a_{i}$ можно редуцировать уравнение Виале к каждому уравнению из списка АБС (см. приложение). Уравнения АБС зависят от двух так называемых решеточных параметров $\alpha$ и $\beta$. 
В терминах функции $Q$ введем для уравнения (1) два многочлена, которые мы будем использовать ниже. Они определяются посредством дискриминантов для $Q$ :

$$
\begin{aligned}
& h\left(u_{0,0}, u_{1,0}\right):=Q \partial_{u_{0,1}} \partial_{u_{1,1}} Q-\partial_{u_{0,1}} Q \partial_{u_{1,1}} Q, \\
& \hat{h}\left(u_{0,0}, u_{0,1}\right):=Q \partial_{u_{1,0}} \partial_{u_{1,1}} Q-\partial_{u_{1,0}} Q \partial_{u_{1,1}} Q .
\end{aligned}
$$

Многочлены $h\left(u_{0,0}, u_{1,0}\right)$ и $\hat{h}\left(u_{0,0}, u_{0,1}\right)$ являются симметричными и биквадратными [19]. В случае уравнений АБС многочлен $h$ можно факторизовать и представить в виде

$$
h(u, x)=k(\alpha, \beta) f(u, x ; \alpha),
$$

где $k$ - кососимметричная функция решеточных параметров, а $f$ - симметричный и биквадратный многочлен по $u$ и $x$ [18]. Многочлены $f(u, x ; \alpha)$ для уравнений АБС перечислены в приложении.

Ковариантность уравнений представляет собой еще одно полезное для нашего изложения свойство. Для уравнения Виале это означает, что оно инвариантно при одновременной замене $u_{1,0} \rightleftarrows u_{0,1}$ и $a_{3} \rightleftarrows a_{5}$. Для всех уравнений АБС ковариантность означает, что мы меняем местами $u_{1,0}$ и $u_{0,1}$, а также решеточные параметры $\alpha$ и $\beta$. Это свойство позволит нам ограничиться изучением симметрий, рекурсионных операторов и законов сохранения только для одного направления решетки (с восстановлением дополнительного набора за счет ковариантности). Будем предполагать, что симметрии и канонические плотности суть элементы из $\mathcal{F}_{\mathbf{s}}$, а рекурсионный оператор есть s-псевдоразностный оператор. Вклад в эти выражения дают только функции и их сдвиги в направлении $\mathcal{S}$. По этой причине ниже мы будем использовать обозначение с одним индексом для сдвигов многочлена $h$, т. е.

$$
h=h\left(u_{0,0}, u_{1,0}\right), \quad h_{i}=\mathcal{S}^{i} h\left(u_{0,0}, u_{1,0}\right),
$$

и для симметрий, например $K_{j}^{(1)}=\mathcal{S}^{j}\left(K^{(1)}\right)$. Индекс "0", означающий отсутствие сдвига функции, мы будем опускать.

Теперь обратимся к анализу симметрий уравнения Виале. Известно [22], [29], что это уравнение допускает обобщенные симметрии $K^{(1)}$ порядка $(-1,1)$, которые имеют вид

$$
K^{(1)}:=\frac{h}{u_{1,0}-u_{-1,0}}-\frac{1}{2} \partial_{u_{1,0}} h=\frac{h_{-1}}{u_{1,0}-u_{-1,0}}+\frac{1}{2} \partial_{u_{-1,0}} h_{-1} .
$$

Используя определение 2 и формулу (8), можно непосредственно проверить следующий результат.

ПреДЛОжЕниЕ 3. Уравнение Виале (1) обладает симметрией порядка $(-2,2)$, которая имеет вид

$$
K^{(2)}=\frac{h h_{-1}}{\left(u_{1,0}-u_{-1,0}\right)^{2}}\left(\frac{1}{u_{2,0}-u_{0,0}}+\frac{1}{u_{0,0}-u_{-2,0}}\right) .
$$

В действительности у уравнения Виале бесконечно много локальных обобщенных симметрий. Иерархии симметрий можно построить, последовательно применяя рекурсионный оператор $\mathfrak{R}$ к затравочным или корневым симметриям $K^{(1)}$ и $K^{(2)}-$ начальным точкам для иерархии симметрий [32]. Коммутативность векторных полей, отвечающих $K^{(1)}$ и $K^{(2)}$, была установлена в работе [33]. 
Теорема 5. Уравнение Виале (1) обладает рекурсионным оператором $\mathfrak{R}=\mathcal{H} \circ \mathcal{I}$, где оператори $\mathcal{H}$ u $\mathcal{I}$ определяются как

$$
\begin{aligned}
\mathcal{I}= & \frac{1}{h} \mathcal{S}-\mathcal{S}^{-1} \frac{1}{h}, \\
\mathcal{H}= & \frac{h_{-1} h h_{1}}{\left(u_{1,0}-u_{-1,0}\right)^{2}\left(u_{2,0}-u_{0,0}\right)^{2}} \mathcal{S}-\mathcal{S}^{-1} \frac{h_{-1} h h_{1}}{\left(u_{1,0}-u_{-1,0}\right)^{2}\left(u_{2,0}-u_{0,0}\right)^{2}}+ \\
& +2 K^{(1)} \mathcal{S}(\mathcal{S}-\mathbf{1})^{-1} \circ K^{(2)}+2 K^{(2)}(\mathcal{S}-\mathbf{1})^{-1} \circ K^{(1)}
\end{aligned}
$$

В теореме 5 операторы $\mathcal{H}$ и $\mathcal{I}$ представляют собой совместные гамильтонов и симплектический операторы для уравнения Виале. Они подробно рассматривались в нашей работе [34], где также было дано полное доказательство теоремы 5 . В той же работе было показано, что как $\mathfrak{R}^{\ell}\left(K^{(1)}\right)$, так и $\mathfrak{R}^{\ell}\left(K^{(2)}\right)$ лежат в $\mathcal{F}_{\mathbf{s}}$ для всех $\ell \in \mathbb{N}$. Это вовсе не очевидно, поскольку гамильтонов оператор $\mathcal{H}$ содержит псевдоразностный оператор $(\mathcal{S}-\mathbf{1})^{-1}$. Симметрии $K^{(1)}$ и $K^{(2)}$ называются затравочными (или корневыми) симметриями для уравнения Виале. Начиная с них можно породить две бесконечные иерархии симметрий, применяя оператор $\mathfrak{R}^{\ell}$. Далее в данном разделе мы дадим доказательство теоремы 5 для частного случая уравнения Н1 (см. ниже теорему 6).

В качестве примера применения полученных результатов мы приведем явное вычисление действия оператора $\mathfrak{R}$ на первую симметрию $K^{(1)}$.

ПреДлОжЕниЕ 4. Уравнение Виале (1) обладает симметрией порядка $(-3,3)$, которая имеет вид

$$
\begin{aligned}
K^{(3)}:=\mathfrak{R}\left(K^{(1)}\right)= & \frac{h h_{-1}}{\left(u_{1,0}-u_{-1,0}\right)^{2}}\left(\frac{K_{2}^{(1)}}{\left(u_{2,0}-u_{0,0}\right)^{2}}+\frac{K_{-2}^{(1)}}{\left(u_{0,0}-u_{-2,0}\right)^{2}}\right)+ \\
& +\left(\frac{1}{u_{2,0}-u_{0,0}}+\frac{1}{u_{0,0}-u_{-2,0}}\right) K^{(1)} K^{(2)} .
\end{aligned}
$$

ДокАзАтЕльство. Для сокращения записи введем обозначение

$$
w:=\frac{1}{u_{1,0}-u_{-1,0}}, \quad w_{k}=\mathcal{S}^{k}(w) .
$$

Сначала рассмотрим произведение операторов $\mathcal{H}$ и $\mathcal{I}$ :

$$
\begin{aligned}
\Re= & h h_{-1} w^{2}\left(w_{1}^{2} \mathcal{S}^{2}+\mathcal{S}^{-2} w_{1}^{2}\right)+2 K^{(1)} K^{(2)}\left(\frac{1}{h} \mathcal{S}+\mathcal{S}^{-1} \frac{1}{h}\right)- \\
& -w^{2}\left(h_{-1} h_{1} w_{1}^{2}+h_{-2} h w_{-1}^{2}\right)+\frac{2}{h_{-1}}\left(K^{(1)} K_{-1}^{(2)}+K^{(2)} K_{-1}^{(1)}\right)+ \\
& +2 K^{(1)}(\mathcal{S}-\mathbf{1})^{-1} \circ\left(\frac{K_{-1}^{(2)}}{h_{-1}}-\frac{K_{1}^{(2)}}{h}\right)+2 K^{(2)}(\mathcal{S}-\mathbf{1})^{-1} \circ\left(\frac{K_{-1}^{(1)}}{h_{-1}}-\frac{K_{1}^{(1)}}{h}\right) .
\end{aligned}
$$

Действуя оператором $\mathfrak{R}$ на симметрию $K^{(1)}$ и используя тождества

$$
K^{(i)} \mathcal{I} K^{(i)}=(\mathcal{S}-\mathbf{1})\left(\frac{1}{h_{-1}} K^{(i)} K_{-1}^{(i)}\right), \quad i=1,2,
$$




$$
\begin{aligned}
K^{(2)} \mathcal{I} K^{(1)}= & (\mathcal{S}-\mathbf{1})\left(\frac{-1}{h} K^{(2)} K_{1}^{(1)}+\frac{1}{2}\left(\frac{1}{u_{2,0}-u_{0,0}}+\frac{1}{u_{0,0}-u_{-2,0}}\right) K^{(2)}\right)+ \\
& +(\mathcal{S}-\mathbf{1})(\mathcal{S}+\mathbf{1})\left(\frac{h h_{-2}}{2\left(u_{1,0}-u_{-1,0}\right)^{2}\left(u_{0,0}-u_{-2,0}\right)^{2}}\right), \\
K^{(1)} \mathcal{I} K^{(2)}= & -K^{(2)} \mathcal{I} K^{(1)}+(\mathcal{S}-\mathbf{1})\left(\frac{1}{h_{-1}} K^{(1)} K_{-1}^{(2)}+\frac{1}{h_{-1}} K^{(2)} K_{-1}^{(1)}\right),
\end{aligned}
$$

получим выражение (44) для симметрии $K^{(3)}$.

Используя приведенные выше тождества, легко вычислить симметрию $K^{(4)}=$ $\mathfrak{R}\left(K^{(2)}\right)$ порядка $(-4,4)$. Применяя $\mathfrak{R}^{N}$ к затравочным симметриям $K^{(1)}$ и $K^{(2)}$, можно породить две иерархии симметрий соответствующих порядков $(-2 N-1,2 N+$ 1) и $(-2 N-2,2 N+2)$ для уравнения Виале.

ЗАмечАниЕ. Симметрии и рекурсионные операторы для уравнений АБС можно получить из симметрий уравнения Виале и оператора $\mathfrak{R}=\mathcal{H} \mathcal{I}$, определенного в теореме 5 , заменяя многочлен $h$ (всюду в симметриях и операторах $\mathcal{I}, \mathcal{H}$ ) на соответствующие многочлены $f:=f\left(u_{0,0}, u_{1,0}, \alpha\right)$. Для всех уравнений АБС многочлены $f$ приведены в приложении. Уравнения Н1-H3, Q1 и Q $3 \delta_{=0}$ допускают точечные симметрии [29]. Для этих уравнений мы нашли дополнительные гамильтоновы операторы нулевого лорановского порядка, которые приведены в приложении.

ПреДЛОЖенИЕ 5. Рекурсионный оператор $\mathfrak{R}$, определенный в теореме 5, для уравнения $\mathrm{H} 1$

$$
\left(u_{0,0}-u_{1,1}\right)\left(u_{1,0}-u_{0,1}\right)=\alpha-\beta
$$

может быть представлен в виде

$$
\mathfrak{R}=\mathfrak{R}_{\mathrm{H} 1}^{2},
$$

где

$$
\mathfrak{R}_{\mathrm{H} 1}=\frac{1}{u_{1,0}-u_{-1,0}}(\mathcal{S}+\mathbf{1})(\mathcal{S}-\mathbf{1})^{-1} \frac{1}{u_{1,0}-u_{-1,0}}\left(\mathcal{S}-\mathcal{S}^{-1}\right) .
$$

ДокАЗАТЕЛьство. В случае уравнения Н1 мы имеем $h=1$. Таким образом, первые две симметрии (41) и (42) соответственно принимают вид

$$
K^{(1)}=\frac{1}{u_{1,0}-u_{-1,0}}, \quad K^{(2)}=\frac{1}{\left(u_{1,0}-u_{-1,0}\right)^{2}}\left(\frac{1}{u_{2,0}-u_{0,0}}+\frac{1}{u_{0,0}-u_{-2,0}}\right) .
$$

Подставляя их в (45), получаем утверждение прямым вычислением.

Оператор, определяемый соотношением (47), является рекурсионным оператором уравнения Н1. Чтобы доказать это, воспользуемся следующим результатом.

Лемма. Onepamop

$$
\begin{aligned}
\mathfrak{M}= & \frac{1}{\left(u_{2,1}-u_{0,1}\right)^{2}} \mathcal{S}+\frac{1}{\left(u_{1,0}-u_{-1,0}\right)^{2}} \mathcal{S}^{-1}+\frac{2}{\left(u_{1,1}-u_{-1,1}\right)\left(u_{2,1}-u_{0,1}\right)}+ \\
& +(\alpha-\beta)\left(\frac{1}{\left(u_{1,1}-u_{-1,1}\right)^{2}\left(u_{1,0}-u_{0,1}\right)^{2}}-\frac{1}{\left(u_{2,1}-u_{0,1}\right)^{2}\left(u_{2,0}-u_{1,1}\right)^{2}}\right)-
\end{aligned}
$$




$$
\begin{aligned}
& -2\left(\frac{1}{u_{1,0}-u_{-1,0}}+\frac{\alpha-\beta}{\left(u_{2,0}-u_{0,0}\right)\left(u_{1,0}-u_{0,1}\right)^{2}}\right) \times \\
& \times(\mathcal{S}-\mathbf{1})^{-1} \circ\left(\frac{1}{u_{2,0}-u_{0,0}}-\frac{u_{1,0}-u_{0,1}}{\left(u_{1,0}-u_{-1,0}\right)\left(u_{0,0}-u_{-1,1}\right)}\right)
\end{aligned}
$$

удовлетворяет следующему тождеству для всех решений уравнения $\mathrm{H} 1$ (46):

$$
\begin{aligned}
& \left(\mathbf{1}+\frac{\alpha-\beta}{\left(u_{1,0}-u_{0,1}\right)^{2}} \mathcal{S}\right) \circ \mathfrak{R}_{\mathrm{H} 1}=\mathfrak{M} \circ\left(\mathbf{1}+\frac{\alpha-\beta}{\left(u_{1,0}-u_{0,1}\right)^{2}} \mathcal{S}\right), \\
& \left(\mathcal{S}+\frac{\alpha-\beta}{\left(u_{1,0}-u_{0,1}\right)^{2}}\right) \circ \mathcal{T}\left(\mathfrak{R}_{\mathrm{H} 1}\right)=\mathfrak{M} \circ\left(\mathcal{S}+\frac{\alpha-\beta}{\left(u_{1,0}-u_{0,1}\right)^{2}}\right) .
\end{aligned}
$$

ДокАзАтЕльство. Доказательство тождеств (49), (50) сводится к непосредственной подстановке явных выражений для $\mathfrak{R}_{\mathrm{H} 1}$ и $\mathfrak{M}$, раскрытию всех скобок, использованию очевидного тождества $\mathcal{S}(\mathcal{S}-1)^{-1}=1+(\mathcal{S}-1)^{-1}$ и применению исключающего отображения $\mathcal{E}$ к результату. Это прямолинейное, но довольно трудоемкое вычисление.

Теорема 6. Оператор $\mathfrak{R}_{\mathrm{H} 1}$, определяемый в (47), является рекурсионным оператором уравнения $\mathrm{H} 1$ (46), причем

$$
\mathfrak{P}=\left(u_{1,0}-u_{0,1}\right) \mathfrak{M} \circ\left(u_{1,0}-u_{0,1}\right)^{-1} \text {. }
$$

ДокАЗАТЕЛЬство. Из теоремы 1 следует, что $\mathfrak{R}_{\mathrm{H} 1}$ является рекурсионным оператором уравнения Н1, откуда следует, что и формулы (16) и (17) справедливы для оператора $\mathfrak{P}$. Перепишем их в виде

$$
\begin{aligned}
\left(Q_{u_{1,0}} \mathcal{S}+Q_{u_{0,0}}\right) \circ \mathfrak{R}_{\mathrm{H} 1} & =\mathfrak{P} \circ\left(Q_{u_{1,0}} \mathcal{S}+Q_{u_{0,0}}\right), \\
\left(Q_{u_{1,1}} \mathcal{S}+Q_{u_{0,1}}\right) \circ \mathcal{T}\left(\mathfrak{R}_{\mathrm{H} 1}\right) & =\mathfrak{P} \circ\left(Q_{u_{1,1}} \mathcal{S}+Q_{u_{0,1}}\right) .
\end{aligned}
$$

Для уравнения Н1 имеем

$$
\begin{aligned}
Q_{u_{1,0}} \mathcal{S}+Q_{u_{0,0}} & =\left(u_{0,0}-u_{1,1}\right) \mathcal{S}+\left(u_{1,0}-u_{0,1}\right)=\left(u_{1,0}-u_{0,1}\right)\left(\mathbf{1}+\frac{\alpha-\beta}{\left(u_{1,0}-u_{0,1}\right)^{2}} \mathcal{S}\right), \\
Q_{u_{1,1}} \mathcal{S}+Q_{u_{0,1}} & =-\left(u_{1,0}-u_{0,1}\right) \mathcal{S}-\left(u_{0,0}-u_{1,1}\right)= \\
& =-\left(u_{1,0}-u_{0,1}\right)\left(\mathcal{S}+\frac{\alpha-\beta}{\left(u_{1,0}-u_{0,1}\right)^{2}}\right) .
\end{aligned}
$$

Используя доказанную выше лемму, легко получить соотношения (52), что и доказывает утверждение теоремы.

Теперь можно воспользоваться результатами п. 3.2, чтобы найти сохраняющиеся плотности для уравнения (1). Если мы непосредственно применим теорему 4 к рекурсионному оператору (45), то поскольку лорановский порядок рекурсионного оператора $\mathfrak{R}$ равен двум, мы получим только “четную" часть бесконечной последовательности канонических законов сохранения. В общем случае квадратный корень разностного формального ряда может не существовать, однако для рекурсионного 
оператора, определенного в теореме 5, он существует, его можно вычислить и таким образом найти формальный рекурсионный оператор первого порядка:

$$
\widetilde{\Re}=\frac{h_{-1}}{\left(u_{1,0}-u_{-1,0}\right)^{2}} \mathcal{S}+\frac{2 K^{(1)}}{u_{0,0}-u_{-2,0}}-\partial_{u_{0,0}} K^{(1)}+\cdots,
$$

для которого $\widetilde{\mathfrak{R}}^{2}=\mathfrak{R}$. Рекурсионный оператор первого порядка дает полный набор канонических законов сохранения, при этом первые три плотности этой последовательности имеют вид

$$
\begin{aligned}
\rho_{0}= & \ln \left(\partial_{u_{1,0}} K^{(1)}\right)=\ln h_{-1}-2 \ln \left(u_{1,0}-u_{-1,0}\right), \\
\rho_{1}= & \frac{2 K^{(1)}}{u_{0,0}-u_{-2,0}}-\partial_{u_{0,0}} K^{(1)} \\
\rho_{2}= & -\frac{1}{\left(u_{1,0}-u_{-1,0}\right)^{2}}\left(\frac{h_{-1} h_{1}}{\left(u_{2,0}-u_{0,0}\right)^{2}}+\frac{h_{-2} h}{\left(u_{0,0}-u_{-2,0}\right)^{2}}\right)+ \\
& +\frac{2}{h_{-1}}\left(K^{(1)} K_{-1}^{(2)}+K^{(2)} K_{-1}^{(1)}\right) .
\end{aligned}
$$

Первая и вторая плотности соответственно представляют собой логарифмический вычет и вычет оператора $\widetilde{\Re}$, а $\rho_{2}$ является вычетом рекурсионного оператора $\mathfrak{R}=$ $\widetilde{\mathfrak{R}}^{2}$, определенного в теореме 5 .

Согласно предложению 1 соответствующие потоки имеют вид

$$
\begin{aligned}
\sigma_{0}= & \mathcal{S}^{-1}\left(\ln \frac{Q_{u_{1,1}}}{Q_{u_{1,0}}}\right) \\
\sigma_{1}= & \mathcal{S}^{-1}\left(\frac{h_{-1}}{\left(u_{1,0}-u_{-1,0}\right)^{2}} F\right), \\
\sigma_{2}= & \mathcal{S}^{-1}\left(\frac{2 F K^{(1)} K^{(2)}}{h}\right)-\mathcal{S}^{-2}\left(\frac{F h h_{-1}}{\left(u_{2,0}-u_{0,0}\right)^{2}\left(u_{1,0}-u_{-1,0}\right)^{2}}\right)- \\
& -\left(1+\mathcal{S}^{-1}\right)\left(\frac{Q_{u_{0}, 0}}{Q_{u_{1,0}}} \frac{h_{-1} h_{-2}}{\left(u_{1,0}-u_{-1,0}\right)^{2}\left(u_{0,0}-u_{-2,0}\right)^{2}} \mathcal{S}^{-1} F\right),
\end{aligned}
$$

где

$$
F=\frac{Q_{u_{0,1}} \mathcal{S}^{-1}\left(Q_{u_{1,0}}\right)}{Q_{u_{1,0}} \mathcal{S}^{-1}\left(Q_{u_{1,1}}\right)}-\frac{Q_{u_{0,0}}}{Q_{u_{1,0}}}
$$

а $Q$ - определяющий многочлен для уравнения (1) (или для уравнений АБС).

Ковариантность уравнения позволяет получить еще три закона сохранения из приведенных выше выражений для плотностей и потоков простой заменой $u_{i, 0}$ на $u_{0, i}, h$ на $\hat{h}$ и оператора $\mathcal{S}$ на $\mathcal{T}$. С другой стороны, эти законы сохранения можно также ограничить на уравнения АБС аналогично тому, как это описано в приведенном выше замечании. 
Рекурсионный оператор (47) для уравнения Н1 является оператором первого порядка, и можно использовать соотношения (31) для построения сохраняющихся плотностей. Для уравнений, допускающих гамильтоновы операторы нулевого лорановского порядка, т. е. для уравнений $\mathrm{H} 1-\mathrm{H} 3, \mathrm{Q} 1$ и $\mathrm{Q} 3_{\delta=0}$, сохраняющиеся плотности можно записать в относительно компактном виде (они эквивалентны формулам, которые приведены в предложении 1):

$$
\begin{aligned}
& \varrho_{0}=\ln \left(\mathcal{S}^{-1} f\right)-2 \ln \left(u_{1,0}-u_{-1,0}\right), \\
& \varrho_{1}=\frac{f}{\left(u_{1,0}-u_{-1,0}\right)\left(u_{2,0}-u_{0,0}\right)}, \\
& \varrho_{2}=\left(\varrho_{1}+\mathcal{S}\left(\varrho_{1}\right)\right)^{2}-\mathcal{S}^{2}\left(\varrho_{1}\right), \\
& \varrho_{3}=\left(\varrho_{1}+\mathcal{S}\left(\varrho_{1}\right)\right)^{3}-\mathcal{S}^{3}\left(\varrho_{1}\right)+3 \varrho_{1} \mathcal{S}\left(\varrho_{1}\right) \mathcal{S}^{2}\left(\varrho_{1}\right), \\
& \varrho_{4}=\left(\varrho_{1}+\mathcal{S}\left(\varrho_{1}\right)\right)^{4}-\mathcal{S}^{4}\left(\varrho_{1}\right)+4 \varrho_{1} \mathcal{S}\left(\varrho_{1}\right) \mathcal{S}^{2}\left(\varrho_{1}\right)\left(\varrho_{1}+2 \mathcal{S}\left(\varrho_{1}\right)+\mathcal{S}^{2}\left(\varrho_{1}\right)+\mathcal{S}^{3}\left(\varrho_{1}\right)\right) .
\end{aligned}
$$

Для уравнения Н1 (46) некоторые из приведенных выше плотностей были получены в работе [24] с помощью метода Гарднера.

\section{5. ЗАКЛЮЧЕНИЕ}

Рекурсионные операторы, представленные в настоящей работе, порождают бесконечные иерархии симметрий и законов сохранения для всех уравнений АБС. Для уравнения Н1 мы показали, что предложенный рекурсионный оператор $\mathfrak{R}_{\mathrm{H} 1}$ действительно удовлетворяет определяющему уравнению (15), и, таким образом, действуя на его симметрию $K^{(1)}$, он будет порождать новую симметрию $\mathfrak{R}_{\mathrm{H} 1}\left(K^{(1)}\right)$, если только такое действие корректно определено (т. е. результат лежит в $\mathcal{F}_{Q}$ ). Доказательство того, что рекурсионный оператор можно применять многократно и каждый раз получать симметрию, требует проверки того, что рекурсионный оператор обладает свойством Нийенхейса [35]-[37], и построения, аналогичного знаменитой схеме Ленарда для уравнения Кортевега-де Фриза [38]. Обобщению схемы Ленарда для разностного уравнения, а также полному доказательству того, что оператор $\mathfrak{R}$, определенный в теореме 5 , действительно удовлетворяет уравнению (15), будет посвящена наша следующая работа.

Для квад-уравнения первое условие интегрируемости было получено Леви и Ямиловым [26]. В настоящей работе мы предложили бесконечную последовательность условий интегрируемости в виде канонических законов сохранения. Условие ЛевиЯмилова представляет собой первый элемент этой последовательности. Мы показали, что канонические законы сохранения являются необходимыми условиями существования формального рекурсионного оператора для разностного уравнения. Как и в случае дифференциальных уравнений в частных производных (ср. [4], [6], [7]), можно доказать, что существование формального рекурсионного оператора следует из существования бесконечной иерархии симметрий возрастающего порядка для разностного уравнения (общему и подробному доказательству этого утверждения будет посвящена отдельная работа). Нет сомнений в том, что условия интегрируемости можно успешно использовать для анализа и классификации интегрируемых разностных уравнений (см., например, [26]).

Нашу теорию можно распространить на разностные уравнения более высоких порядков, т. е. на уравнения на прямоугольнике с функцией $Q$, зависящей от набора 
переменных $\left\{u_{p, q} \mid 0 \leqslant p \leqslant N, 0 \leqslant q \leqslant M\right\}$. Для существования отображения исключения $\mathcal{E}$ следует предположить, что уравнение $Q=0$ можно единственным образом разрешить относительно угловых переменных $u_{0,0}, u_{N, 0}, u_{0, M}$ и $u_{N, M}$. В этом случае динамические переменные можно определить как

$U_{\mathbf{s}}=\left\{u_{n, p} \mid n \in \mathbb{Z}, 0 \leqslant p<M\right\}, \quad U_{\mathbf{t}}=\left\{u_{q, m} \mid m \in \mathbb{Z}, 0 \leqslant q<N\right\}, \quad U_{0}=U_{\mathbf{s}} \cup U_{\mathbf{t}}$.

Мы работаем над обобщением нашей теории на случай более высоких порядков, векторный случай, а также случай, когда не предполагается, что $Q$ является аффиннолинейным многочленом.

\section{Уравнения АБС}

ПРИЛОЖЕНИЕ

Для каждого уравнения в классификации АБС [18] мы приведем здесь определяющий многочлен $Q$, соответствующий выбор для параметров $a_{i}$ из $(1)$, соответствующие многочлен $f$ и множитель $k$ из (40), гамильтонов оператор $\mathcal{H}$ и затравочную (корневую) симметрию иерархии. Уравнение Q4, известное как уравнение Адлера, мы приводим в виде, взятом из работы [39].

Рекурсионный оператор для всех уравнений АБС можно записать как $\mathfrak{R}=\mathcal{H} \circ \mathcal{I}$, где симплектический оператор $\mathcal{I}$ имеет вид

$$
\mathcal{I}=\frac{1}{f} \mathcal{S}-\mathcal{S}^{-1} \frac{1}{f}
$$

а гамильтонов оператор $\mathcal{H}$ (для всех уравнений АБС) имеет вид

$$
\begin{aligned}
\mathcal{H}= & \frac{f_{-1} f f_{1}}{\left(u_{1,0}-u_{-1,0}\right)^{2}\left(u_{2,0}-u_{0,0}\right)^{2}} \mathcal{S}-\mathcal{S}^{-1} \frac{f_{-1} f f_{1}}{\left(u_{1,0}-u_{-1,0}\right)^{2}\left(u_{2,0}-u_{0,0}\right)^{2}}+ \\
& +2\left(K^{(1)} \mathcal{S}(\mathcal{S}-\mathbf{1})^{-1} K^{(2)}+K^{(2)}(\mathcal{S}-\mathbf{1})^{-1} K^{(1)}\right) .
\end{aligned}
$$

Для уравнений $\mathrm{H} 1-\mathrm{H} 3, \mathrm{Q} 1$ и $\mathrm{Q} 3_{\delta=0}$ имеется более простой гамильтонов оператор, приведенный ниже. В равенстве $(55)$ и ниже $K^{(1)}$ и $K^{(2)}$ обозначают первые симметрии уравнений АБС, которые имеют вид

$$
\begin{aligned}
& K^{(1)}=\frac{f}{u_{1,0}-u_{-1,0}}-\frac{1}{2} \partial_{u_{1,0}} f=\frac{f_{-1}}{u_{1,0}-u_{-1,0}}+\frac{1}{2} \partial_{u_{-1,0}} f_{-1}, \\
& K^{(2)}=\frac{f f_{-1}}{\left(u_{1,0}-u_{-1,0}\right)^{2}}\left(\frac{1}{u_{2,0}-u_{0,0}}+\frac{1}{u_{0,0}-u_{-2,0}}\right) .
\end{aligned}
$$

Уравнение Н1:

уравнение

параметры

многочлен $h=k \cdot f$

оператор $\mathcal{H}$

затравочная симметрия

$$
Q=\left(u_{0,0}-u_{1,1}\right)\left(u_{1,0}-u_{0,1}\right)-\alpha+\beta
$$

$a_{3}=-a_{5}=1, a_{7}=\beta-\alpha$,

$a_{1}=a_{2}=a_{4}=a_{6}=0$;

$f=1, k=\beta-\alpha$;

$\mathcal{H}=K^{(1)}(\mathcal{S}+\mathbf{1})(\mathcal{S}-\mathbf{1})^{-1} K^{(1)} ;$

$K^{(1)}$. 
Уравнение Н2:

уравнение

параметры

многочлен $h=k \cdot f$

оператор $\mathcal{H}$

затравочная симметрия

уравнение

параметры

многочлен $h=k \cdot f$

оператор $\mathcal{H}$

затравочная симметрия

уравнение

параметры

многочлен $h=k \cdot f$

оператор $\mathcal{H}$

затравочная симметрия

уравнение

параметры

многочлен $h=k \cdot f$

оператор $\mathcal{H}$

затравочные симметрии

$$
\begin{aligned}
Q= & \left(u_{0,0}-u_{1,1}\right)\left(u_{1,0}-u_{0,1}\right)+(\beta-\alpha)\left(u_{0,0}+\right. \\
& \left.+u_{1,0}+u_{0,1}+u_{1,1}\right)-\alpha^{2}+\beta^{2}
\end{aligned}
$$

$a_{3}=-a_{5}=1, a_{6}=\beta-\alpha$,

$a_{7}=\beta^{2}-\alpha^{2}, a_{1}=a_{2}=a_{4}=0$;

$f=2\left(u_{0,0}+u_{1,0}+\alpha\right), k=\beta-\alpha$;

$\mathcal{H}=K^{(1)}(\mathcal{S}+\mathbf{1})(\mathcal{S}-\mathbf{1})^{-1} K^{(1)}-(\mathcal{S}-\mathbf{1})(\mathcal{S}+\mathbf{1})^{-1}$;

$K^{(1)}$.

\section{Уравнение Н3:}

$Q=\alpha\left(u_{0,0} u_{1,0}+u_{0,1} u_{1,1}\right)-$

$$
-\beta\left(u_{0,0} u_{0,1}+u_{1,0} u_{1,1}\right)+\delta\left(\alpha^{2}-\beta^{2}\right) ;
$$

$a_{3}=\alpha, a_{5}=-\beta, a_{7}=\delta\left(\alpha^{2}-\beta^{2}\right)$,

$a_{1}=a_{2}=a_{4}=a_{6}=0$;

$f=u_{0,0} u_{1,0}+\alpha \delta, k=\alpha^{2}-\beta^{2}$;

$\mathcal{H}=K^{(1)}(\mathcal{S}+\mathbf{1})(\mathcal{S}-\mathbf{1})^{-1} K^{(1)}-$

$-(1 / 4) u_{0,0}(\mathcal{S}-\mathbf{1})(\mathcal{S}+\mathbf{1})^{-1} u_{0,0}$

$K^{(1)}$.

Уравнение Q1:

$Q=\alpha\left(u_{0,0}-u_{0,1}\right)\left(u_{1,0}-u_{1,1}\right)-$

$$
-\beta\left(u_{0,0}-u_{1,0}\right)\left(u_{0,1}-u_{1,1}\right)+\delta^{2} \alpha \beta(\alpha-\beta) ;
$$

$a_{3}=\alpha, a_{4}=\beta-\alpha, a_{5}=-\beta$,

$a_{7}=\delta^{2} \alpha \beta(\alpha-\beta), a_{1}=a_{2}=a_{6}=0$;

$f=\left(\left(u_{0,0}-u_{1,0}\right)^{2}-\alpha^{2} \delta^{2}\right) / \alpha, \quad k=\alpha \beta(\beta-\alpha)$;

$\mathcal{H}=K^{(1)}(\mathcal{S}+\mathbf{1})(\mathcal{S}-\mathbf{1})^{-1} K^{(1)}-$

$$
-\delta^{2}(\mathcal{S}+\mathbf{1})(\mathcal{S}-\mathbf{1})^{-1}
$$

$K^{(1)}$.

Уравнение Q2:

$$
\begin{aligned}
Q= & \alpha\left(u_{0,0}-u_{0,1}\right)\left(u_{1,0}-u_{1,1}\right)- \\
& -\beta\left(u_{0,0}-u_{1,0}\right)\left(u_{0,1}-u_{1,1}\right)+ \\
& +\alpha \beta(\alpha-\beta)\left(u_{0,0}+u_{1,0}+u_{0,1}+u_{1,1}\right)- \\
& -\alpha \beta(\alpha-\beta)\left(\alpha^{2}-\alpha \beta+\beta^{2}\right) ;
\end{aligned}
$$

$a_{3}=\alpha, a_{4}=\beta-\alpha, a_{5}=-\beta$,

$a_{6}=\alpha \beta(\alpha-\beta)$,

$a_{7}=\alpha \beta(\beta-\alpha)\left(\alpha^{2}-\alpha \beta+\beta^{2}\right), a_{1}=a_{2}=0 ;$

$f=\left(\left(u_{0,0}-u_{1,0}\right)^{2}-2 \alpha^{2}\left(u_{0,0}+u_{1,0}\right)+\alpha^{4}\right) / \alpha$,

$k=\alpha \beta(\beta-\alpha)$;

задается формулой (55);

$K^{(1)}, K^{(2)}$. 
Уравнение Q3:

уравнение

$$
\begin{aligned}
Q= & \left(\beta^{2}-\alpha^{2}\right)\left(u_{0,0} u_{1,1}+u_{1,0} u_{0,1}\right)+ \\
& +\beta\left(\alpha^{2}-1\right)\left(u_{0,0} u_{1,0}+u_{0,1} u_{1,1}\right)- \\
& -\alpha\left(\beta^{2}-1\right)\left(u_{0,0} u_{0,1}+u_{1,0} u_{1,1}\right)- \\
& -\delta^{2}\left(\alpha^{2}-\beta^{2}\right)\left(\alpha^{2}-1\right)\left(\beta^{2}-1\right) / 4 \alpha \beta ;
\end{aligned}
$$

параметры

$$
\begin{aligned}
& a_{3}=\beta\left(\alpha^{2}-1\right), a_{4}=\beta^{2}-\alpha^{2}, a_{5}=-\alpha\left(\beta^{2}-1\right), \\
& a_{7}=-\delta^{2}\left(\alpha^{2}-\beta^{2}\right)\left(\alpha^{2}-1\right)\left(\beta^{2}-1\right) / 4 \alpha \beta, \\
& a_{1}=a_{2}=a_{6}=0 ;
\end{aligned}
$$

многочлен $h=k \cdot f$

$$
\begin{aligned}
f= & \left(4 \alpha\left(\alpha^{2}-1\right)\right)^{-1}\left(4 \alpha\left(\alpha u_{0,0}-u_{1,0}\right)\left(\alpha u_{1,0}-u_{0,0}\right)-\right. \\
& \left.-\left(\alpha^{2}-1\right)^{2} \delta^{2}\right), \\
k= & \left(\alpha^{2}-\beta^{2}\right)\left(\alpha^{2}-1\right)\left(\beta^{2}-1\right) ;
\end{aligned}
$$

оператор $\mathcal{H}$

1) $\delta=0: \mathcal{H}=K^{(1)}(\mathcal{S}+\mathbf{1})(\mathcal{S}-\mathbf{1})^{-1} K^{(1)}-$

$$
-(1 / 4) u_{0,0}(\mathcal{S}+\mathbf{1})(\mathcal{S}-\mathbf{1})^{-1} u_{0,0},
$$

2) $\delta \neq 0$ : задается формулой (55);

затравочные симметрии

1) $\left.\delta=0: K^{(1)}, 2\right) \delta \neq 0: K^{(1)}, K^{(2)}$.

\section{Уравнение Q4:}

уравнение

$$
\begin{aligned}
Q= & \operatorname{sn} \alpha\left(u_{0,0} u_{1,0}+u_{0,1} u_{1,1}\right)- \\
& -\operatorname{sn} \beta\left(u_{0,0} u_{0,1}+u_{1,0} u_{1,1}\right)- \\
& -\operatorname{sn}(\alpha-\beta)\left(u_{0,0} u_{1,1}+u_{1,0} u_{0,1}\right)+ \\
& +k \operatorname{sn} \alpha \operatorname{sn} \beta \operatorname{sn}(\alpha-\beta)\left(1+u_{0,0} u_{1,0} u_{0,1} u_{1,1}\right) ;
\end{aligned}
$$

параметры

$$
a_{1}=a_{7}=k \operatorname{sn} \alpha \operatorname{sn} \beta \operatorname{sn}(\alpha-\beta), a_{3}=\operatorname{sn} \alpha \text {, }
$$$$
a_{4}=-\operatorname{sn}(\alpha-\beta), a_{5}=-\operatorname{sn} \beta, a_{2}=a_{6}=0 \text {; }
$$

многочлен $h=k \cdot f$

$$
\begin{aligned}
f= & -k \operatorname{sn} \alpha\left(1+u_{0,0}^{2} u_{1,0}^{2}\right)+(\operatorname{sn} \alpha)^{-1}\left(u_{0,0}^{2}+u_{1,0}^{2}-\right. \\
& \left.\quad-2 u_{0,0} u_{1,0} \operatorname{cn} \alpha \operatorname{dn} \alpha\right), \\
k= & \operatorname{sn} \alpha \operatorname{sn} \beta \operatorname{sn}(\beta-\alpha) ;
\end{aligned}
$$

оператор $\mathcal{H}$

задается формулой (55);

затравочные симметрии

$$
K^{(1)}, K^{(2)} \text {. }
$$

Благодарности. Авторы благодарны В. Е. Адлеру за разъяснения, касающиеся уравнения Виале, а также К. Асорну, К.С. Гао, Е. Хуберту, А. Пилай и С. Юань за полезные обсуждения. А. В. Михайлов и П. Ксенитидис благодарят Университет Кента за гостеприимство. Дж. П. Ванг благодарит Университет Кента за поддержку научного отпуска. Работа П. Ксенитидиса выполнена при финансовой поддержке Международной Ньютоновской стипендии (грант NF082473 “Симметрии и интегрируемость решеточных уравнений и связанных с ними дифференциальных уравнений в частных производных"). 
А. В. МИХАЙЛОВ, ДЖ.П. ВАНГ, П. КСЕНИТИДИС

\section{Список литературы}

[1] V.E. Zakharov (ed.), What is Integrability?, Springer Ser. Nonlinear Dynam., Springer, Berlin, 1991.

[2] A. V. Mikhailov (ed.), Integrability, Lecture Notes in Phys., 767, Springer, Berlin, 2009.

[3] П. Олвер, Приложения групп Ли к дифференииальным уравнениям, Мир, М., 1989.

[4] V. V. Sokolov, A. B. Shabat, "Classification of integrable evolution equations", Mathematical Physics Reviews, v. 4, Sov. Sci. Rev., Sect. C, 4, ed. S. P. Novikov, Harwood Academic, Chur, 1984, 221-280.

[5] A. V. Mikhailov, A. B. Shabat, R. I. Yamilov, Comm. Math. Phys., 115:1 (1988), 1-19.

[6] А. В. Михайлов, А. Б. Шабат, Р. И. Ямилов, УМН, 42:4(256) (1987), 3-53.

[7] А. В. Михайлов, А. Б. Шабат, В.В.Соколов, “Симметрийный подход к классификации интегрируемых уравнений", Интегрируемость и кинетические уравнения для солитонов, ред. В. Г. Бахтарьяр, В. Е. Захаров, В. М. Черноусенко, Наукова думка, Киев, 1990, 213-279.

[8] J. A. Sanders, J. P. Wang, J. Differ. Equations, 147:2 (1998), 410-434.

[9] J.P. Wang, Symmetries and conservation laws of evolution equations, PhD thesis, Vrije Universiteit/Thomas Stieltjes Institute, Amsterdam, 1998.

[10] В.Э. Адлер, А. Б. Шабат, Р. И. Ямилов, ТМФ, 125:3 (2000), 355-424.

[11] Р. И. Ямилов, УМН, 38:6 (1983), 155-156.

[12] R. I. Yamilov, J. Phys. A, 39:45 (2006), R541-R623.

[13] F. W. Nijhoff, H. W. Capel, Acta Appl. Math., 39:1-3 (1995), 133-158.

[14] B. Grammaticos, R. G. Halburd, A. Ramani, C.-M. Viallet, J. Phys. A, 42:45 (2009), 454002, $30 \mathrm{pp}$.

[15] A. I. Bobenko, Yu. B. Suris, Int. Math. Res. Not., 2002:11 (2002), 573-611, arXiv: nlin/0110004.

[16] F. W. Nijhoff, Phys. Lett. A, 297:1-2 (2002), 49-58, arXiv: nlin/0110027.

[17] F. W. Nijhoff, A. J. Walker, Glasg. Math. J., 43:A (2001), 109-123.

[18] V. E. Adler, A. I. Bobenko, Yu. B. Suris, Comm. Math. Phys., 233:3 (2003), 513-543, arXiv: nlin/0202024.

[19] В. Э. Адлер, А. И. Бобенко, Ю. Б. Сурис, Функи. анализ и его прил., 43:1 (2009), 3-21.

[20] M.P. Bellon, C.-M. Viallet, Comm. Math. Phys., 204:2 (1999), 425-437, arXiv: chao-dyn/9805006.

[21] C. M. Viallet, Glasg. Math. J., 51:A (2009), 157-163, arXiv: 0802.0294.

[22] P. Xenitidis, Integrability and symmetries of difference equations: the Adler-Bobenko-Suris case, arXiv: 0902.3954.

[23] O. G. Rasin, P. E. Hydon, J. Phys. A, 40:42 (2007), 12763-12773.

[24] A. G. Rasin, J. Schiff, J. Phys. A, 42:17 (2009), 175205, 16 pp., arXiv: 0901.0390.

[25] A. G. Rasin, J. Phys. A, 43:23 (2010), 235201, 11 pp., arXiv: 1001.0724.

[26] D. Levi, R. I. Yamilov, J. Phys. A, 42:45 (2009), 454012, 18 pp., arXiv: 0902.4421.

[27] G.-L. Zhang, X.-S. Gao, "Properties of ascending chains for partial difference polynomial systems", Lecture Notes in Artificial Intelligence, Lecture Notes in Comput. Sci., 5081, ed. D. Kapur, Springer, Berlin, 2008, 307-321.

[28] V. G. Papageorgiou, F. W. Nijhoff, H. W. Capel, Phys. Lett. A, 147:2-3 (1990), 106-114.

[29] A. Tongas, D. Tsoubelis, P. Xenitidis, J. Phys. A, 40:44 (2007), 13353-13384, arXiv: 0707.3730.

[30] А. В. Жибер, В. В. Соколов, УМН, 56:1(337) (2001), 63-106.

[31] M. Adler, Invent. Math., 50:3 (1978/79), 219-248.

[32] J. A. Sanders, J. P. Wang, Nonlin. Anal., 47:8 (2001), 5213-5240.

[33] A. B. Shabat, R. I. Yamilov, Phys. Lett. A, 130:4-5 (1988), 271-275. 
[34] A. V. Mikhailov, J. P. Wang, P. Xenitidis, Cosymmetries and Nijenhuis recursion operators for difference equations, arXiv: 1009.2403.

[35] И. М. Гельфанд, И. Я. Дорфман, Функи. анализ и его прил., 13:4 (1979), 13-30.

[36] A. S. Fokas, B. Fuchssteiner, Lett. Nuovo Cimento (2), 28:8 (1980), 299-303.

[37] I. Ya. Dorfman, Dirac Structures and Integrability of Nonlinear Evolution Equations, Nonlin. Sci.: Theory and Appl., John Wiley and Sons, Chichester, 1993.

[38] J. P. Wang, J. Math. Phys., 50:2 (2009), 023506, 25 pp., arXiv: 0809.3899.

[39] J. Hietarinta, J. Nonlin. Math. Phys., 12, Suppl. 2 (2005), 223-230.

Поступила в редакцию 15.11.2010 